\title{
Complete genome sequence and comparative genomic analyses of the vancomycin-producing Amycolatopsis orientalis
}

$\mathrm{Li} \mathrm{Xu}^{1,2+}$, He Huang ${ }^{3 \dagger}$, Wei Wei ${ }^{2 \dagger}$, Yi Zhong ${ }^{3,10}$, Biao Tang ${ }^{4}$, Hua Yuan ${ }^{3}$, Li Zhu ${ }^{2}$, Weiyi Huang ${ }^{1}$, Mei Ge ${ }^{2}$, Shen Yang ${ }^{3}$, Huajun Zheng ${ }^{5}$, Weihong Jiang ${ }^{3^{*}}$, Daijie Chen ${ }^{2,6^{*}}$, Guo-Ping Zhao ${ }^{3,4,5,7^{*}}$ and Wei Zhao ${ }^{3,4,8,9^{*}}$

\begin{abstract}
Background: Amycolatopsis orientalis is the type species of the genus and its industrial strain HCCB10007, derived from ATCC 43491, has been used for large-scale production of the vital antibiotic vancomycin. However, to date, neither the complete genomic sequence of this species nor a systemic characterization of the vancomycin biosynthesis cluster $(\mathrm{vcm})$ has been reported. With only the whole genome sequence of Amycolatopsis mediterranei available, additional complete genomes of other species may facilitate intra-generic comparative analysis of the genus.

Results: The complete genome of A. orientalis HCCB10007 comprises an 8,948,591-bp circular chromosome and a 33,499-bp dissociated plasmid. In total, 8,121 protein-coding sequences were predicted, and the species-specific genomic features of $A$. orientalis were analyzed in comparison with that of $A$. mediterranei. The common characteristics of Amycolatopsis genomes were revealed via intra- and inter-generic comparative genomic analyses within the domain of actinomycetes, and led directly to the development of sequence-based Amycolatopsis molecular chemotaxonomic characteristics (MCCs). The chromosomal core/quasi-core and non-core configurations of the A. orientalis and the A. mediterranei genome were analyzed reciprocally, with respect to further understanding both the discriminable criteria and the evolutionary implementation. In addition, 26 gene clusters related to secondary metabolism, including the 64-kb vcm cluster, were identified in the genome. Employing a customized PCR-targeting-based mutagenesis system along with the biochemical identification of vancomycin variants produced by the mutants, we were able to experimentally characterize a halogenase, a methyltransferase and two glycosyltransferases encoded in the vcm cluster. The broad substrate spectra characteristics of these modification enzymes were inferred.
\end{abstract}

Conclusions: This study not only extended the genetic knowledge of the genus Amycolatopsis and the biochemical knowledge of vcm-related post-assembly tailoring enzymes, but also developed methodology useful for in vivo studies in A. orientalis, which has been widely considered as a barrier in this field.

Keywords: Amycolatopsis orientalis, Complete genome sequencing, Molecular taxonomic characteristics, Vancomycin biosynthesis

\footnotetext{
*Correspondence: whjiang@sibs.ac.cn; hccb001@163.com; gpzhao@sibs.ac.cn;

181zhao@163.com

†Equal contributors

${ }^{3}$ CAS Key Laboratory of Synthetic Biology, Institute of Plant Physiology and

Ecology, Shanghai Institutes for Biological Sciences, Chinese Academy of

Sciences, Shanghai 200031, China

${ }^{2}$ Shanghai Laiyi Center for Biopharmaceutical R\&D, Shanghai 200240, China

Full list of author information is available at the end of the article
}

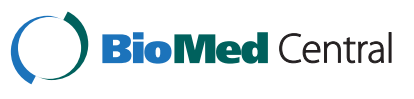

(C) 2014 Xu et al.; licensee BioMed Central Ltd. This is an Open Access article distributed under the terms of the Creative Commons Attribution License (http://creativecommons.org/licenses/by/2.0), which permits unrestricted use, distribution, and reproduction in any medium, provided the original work is properly credited. The Creative Commons Public Domain Dedication waiver (http://creativecommons.org/publicdomain/zero/1.0/) applies to the data made available in this article, unless otherwise stated. 


\section{Background}

Amycolatopsis orientalis is a Gram-positive filamentous actinomycete that produces vancomycin (Figure 1), which is a potent glycopeptide antibiotic that has been used for more than three decades for the treatment of serious methicillin-resistant Staphylococcus aureus (MRSA) infections [1]. However, the reports of increased emergence of vancomycin-resistant $S$. aureus (VRSA) and vancomycinresistant enterococci (VRE) in recent years have presented an urgent challenge to human health, which requires the development of new antibiotics against these pathogens [2-5]. Although some semisynthetic lipoglycopeptide antibiotics, such as telavancin, oritavancin and dalbavancin have been developed recently and their anti-VRSA activities proved in vitro [6], the in vivo potency of these antibiotics is yet to be demonstrated specifically by clinical studies. Thus, further discovery and development of new glycopeptide type drug candidates continues to be an important mission for biologists and organic chemists.

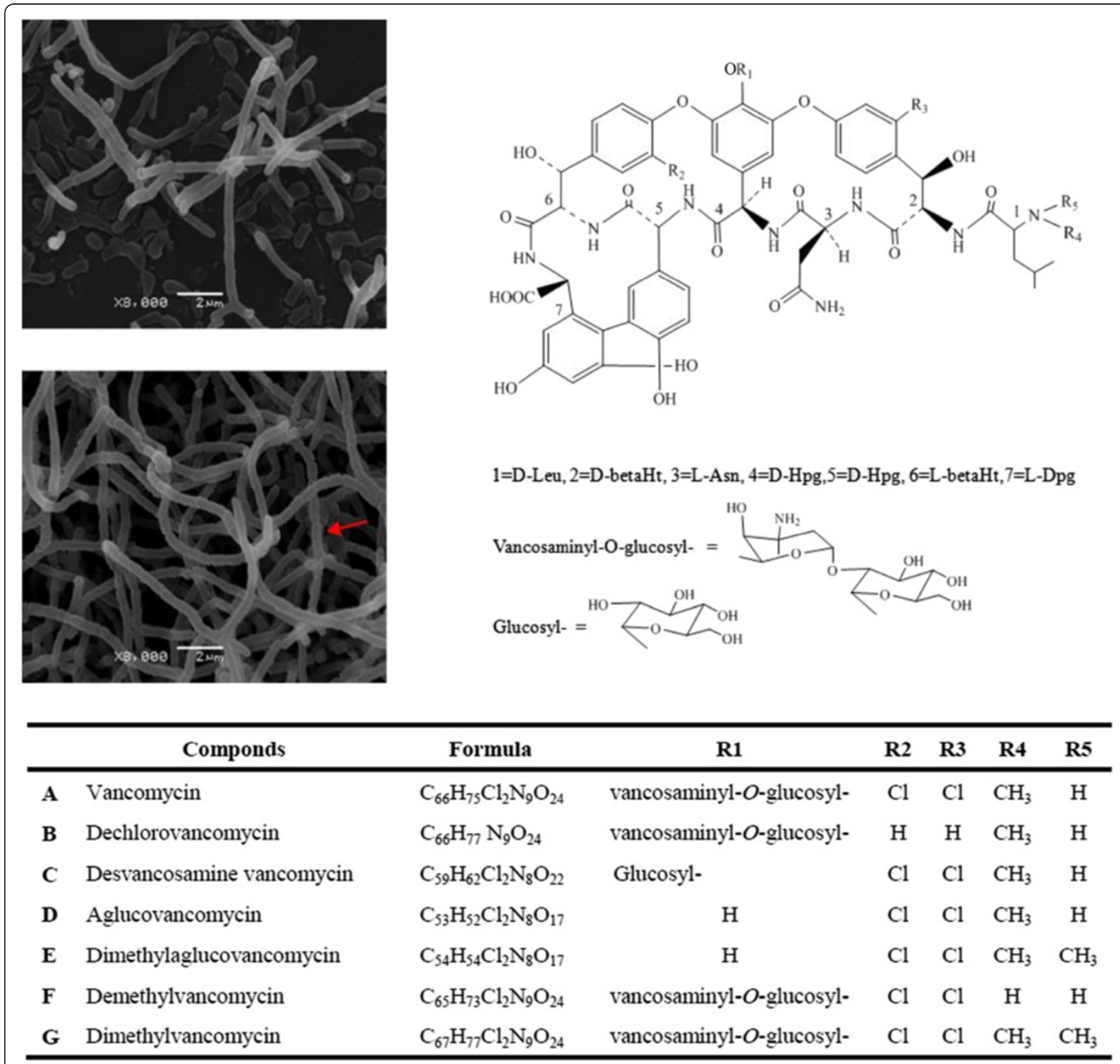

Figure 1 Morphological differentiation of mycelia in Amycolatopsis orientalis HCCB10007 and chemical structures of vancomycin variants. Scanning electron micrograph of A. orientalis HCCB10007 cultured for one or three incubation days (upper left of the panel). The red arrow indicates the sporulation of A. orientalis cultured for three days. The core structural formula proposed for vancomycin and its variants (upper right of the panel) shows minor modifications of the heptapeptide core of vancomycin. Table below shows the specific formulae and radical compositions of each vancomycin variant compounds. Alphabetic numbering in the table are corresponding to the legend of Figure 6 . 
The gene clusters responsible for the biosynthesis of chloroeremomycin (cep) in A. orientalis A82846 and balhimycin (bal) in Amycolatopsis balhimycina DSM 5908, both of which possess an identical heptapeptide backbone and similar antibiotic activities compared with vancomycin, were completely (cep, NCBI accession number: AJ223998, AJ223999, and AL078635) or partially (bal, NCBI accession number: Y16952) sequenced and annotated about 10 years ago $[7,8]$. Thereafter, a series of genes from the two clusters, especially those encoding the postassembly tailoring enzymes involved in chlorination [9], glycosylation [10-13], and methylation [14], were characterized sequentially. For example, the crystal structures of the TDP-epi-vancosaminyltransferase, GtfA [12]; the UDP-glucosyltransferase, GtfB [11]; and the glycopeptide N-methyltransferase, MtfA [14] from A. orientalis A82846 were resolved. The methylation function of MtfA from $A$. orientalis A82846 in the synthesis of glycopeptide antibiotics was studied in Streptomyces toyocaensis [14], and the halogenase activity of BhaA from A. balhimycina DSM 5908 was verified in vivo [9]. Of the enzymes encoded by the biosynthetic gene cluster of vancomycin $(\mathrm{vcm})$ in A. orientalis ATCC 43491, in vitro experiments demonstrated that GtfE is responsible for the addition of $\mathrm{D}$-glucose to the hydroxyl of 4-hydroxyphenylglycine and that GtfD can transfer the L-vancosamine moiety to variant glucosyl-peptides as its substrates $[10,15]$. In fact, as early as 1997, the A. orientalis GtfE was expressed in S. toyocaensis, and a hybrid glycopeptide antibiotic, namely glucosyl A47934, was produced [16]. However, unlike A. balhimycina, A. orientalis is not amenable for genetic manipulations because of difficulties encountered in DNA transformation [17]. Therefore, most genes of the $1 \mathrm{~cm}$ cluster have been characterized by heterologous expression or in vitro enzymatic/structural analysis $[15,18,19]$, with little in vivo data reported.

To date, the DNA sequences, along with their annotation information, have been provided for $v \mathrm{~cm}$ cluster genes including those encoding the monooxygenases (NCBI accession number: AF486630.1, FJ532347.1), the halogenase (NCBI accession number: FJ532347.1), the glycosyltransferases (NCBI accession number: U84350.1) and the vancomycin-resistance proteins (NCBI accession number: AF060799.1). Of these, the functions of the monooxygenase (OxyB [19]), glycosyltransferases (GtfE and GtfD $[10,15,16])$, and the vancomycin-resistance proteins (VanHAX $[20,21]$ ) have been well characterized. We cloned and sequenced the whole $v \mathrm{~cm}$ gene cluster in 2010 (NCBI accession number: HQ679900.1). However, with the exception of the glycosyltransferases and their encoding genes $[10,15]$, other post-assembly tailoring enzymes encoded in the $v \mathrm{~cm}$ cluster, such as the halogenase and the methyltransferase, have been barely experimentally characterized so far and their functions are only assumed based on the similarity of the proteins to those encoded by bal or cep $[9,14]$.

The complete genome sequences of the rifamycin producers Amycolatopsis mediterranei [22,23] not only revealed the special genomic features of the genus Amycolatopsis, but also confirmed it as a clade of rare actinomycetes potentially rich in antibiotic production capabilities. However, although three draft datasets for the genomes of A. orientalis subsp. orientalis were released recently [22,23], neither the annotation nor genomic analysis for these glycopeptide antibiotic-producing Amycolatopsis strains is available to date, particularly, at the level of the complete genome sequence. Here, we report the whole genome sequence of an industrial strain (HCCB10007) of $A$. orientalis (CP003410 and CP003411). This strain produces high yields of vancomycin, and is derived from the species type strain ATCC 43491 through series of physical and chemical mutageneses. The high-quality complete genome sequence of $A$. orientalis was compared intra- and inter-generically to those of its close or distant phylogenetic relatives within the domain of actinomycetes to characterize species-specific and genuscommon features of the genomes. Moreover, functions of the predicted halogenase and methyltransferase of the $v \mathrm{~cm}$ cluster in $A$. orientalis were characterized via robust spectroscopic analyses in the corresponding site-specific mutants, generated by a customized homologous recombination mutation method.

\section{Results and discussion}

\section{General and species-specific features of the complete A. orientalis genome}

The genome of $A$. orientalis HCCB10007 comprises two replicons (Figure 2), a large circular chromosome (8,948,591 bp) and a small, dissociated circular plasmid (33,499 bp). The same circular chromosomal topology with that of $A$. mediterranei U32 [24] and A. mediterranei S699 [25,26], which are the other two complete genomes of the Amycolatopsis genus currently available, implies that this is a common topological feature that differs from the Streptomyces linear chromosomes [27]. The genome of A. orientalis HCCB10007 is much smaller $(1.3 \mathrm{Mbp})$ than that of $A$. mediterranei, and only 8,121 protein-coding sequences (CDSs) were predicted, which is approximately 1,100 fewer CDSs than those identified in the genome of $A$. mediterranei (Table 1 ). The difference is mainly accounted for $\sim 1.1 \mathrm{Mbp}$ shorter in the length of the non-core regions of $A$. orientalis. Furthermore, this difference is also enhanced to a certain extent (about $0.2 \mathrm{Mbp}$ ) by the smaller average size of the intergenic region (IR) both in the core and the non-core regions of the $A$. orientalis genome (Table 1), resulting in a more compact arrangement of genes (coding density of 90.4\%) compared with that of A. mediterranei (89.1-89.3\%). 


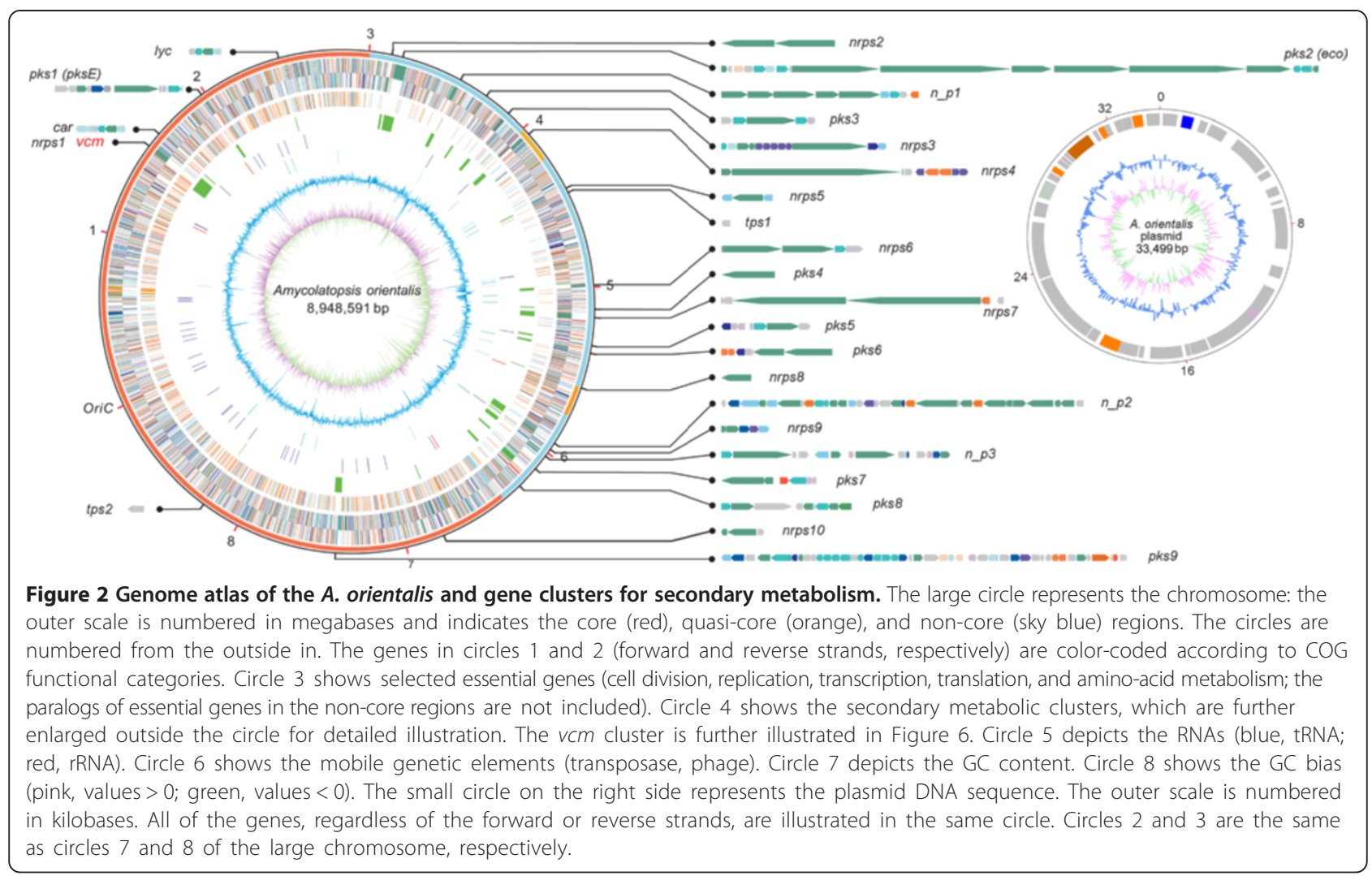

Table 1 General features of the Amycolatopsis genomes

\begin{tabular}{|c|c|c|c|c|c|c|c|}
\hline \multirow{2}{*}{$\begin{array}{l}\text { Species } \\
\text { Strain }\end{array}$} & & \multirow{2}{*}{\multicolumn{2}{|c|}{$\begin{array}{l}\text { A. orientalis } \\
\text { HCCB10007 }\end{array}$}} & \multicolumn{4}{|c|}{ A. mediterranei } \\
\hline & & & & \multicolumn{2}{|c|}{ U32 } & \multicolumn{2}{|c|}{ S699 } \\
\hline \multicolumn{2}{|l|}{ Length (bp) } & \multicolumn{2}{|c|}{$8,948,591$} & \multicolumn{2}{|c|}{$10,236,715$} & \multicolumn{2}{|c|}{$10,246,920$} \\
\hline \multicolumn{2}{|l|}{ Core/quasi-core vs Noncore (Mbp) } & 5.9 & 3.0 & 6.1 & 4.1 & 6.1 & 4.1 \\
\hline \multicolumn{2}{|l|}{ GC content } & \multicolumn{2}{|c|}{$69.0 \%$} & \multicolumn{2}{|c|}{$71.3 \%$} & \multicolumn{2}{|c|}{$71.3 \%$} \\
\hline \multicolumn{2}{|l|}{ Core/quasi-core vs Noncore } & $69.0 \%$ & $69.0 \%$ & $71.1 \%$ & $71.6 \%$ & $71.1 \%$ & $71.6 \%$ \\
\hline ORFs & Total & \multicolumn{2}{|c|}{8,121} & \multicolumn{2}{|c|}{9,228} & \multicolumn{2}{|c|}{9,227} \\
\hline \multirow[t]{4}{*}{ Core/quasi-core vs Noncore } & & 5,624 & 2,497 & 5,627 & 3,601 & 5,630 & 3,597 \\
\hline & Proteins with function assigned & \multicolumn{2}{|c|}{$5,518(67.9 \%)$} & \multicolumn{2}{|c|}{$6,441(69.8 \%)$} & \multicolumn{2}{|c|}{$6,386(69.2 \%)$} \\
\hline & Hypothetical proteins with unknown function & \multicolumn{2}{|c|}{$2,603(32.1 \%)$} & \multicolumn{2}{|c|}{$2,787(30.2 \%)$} & \multicolumn{2}{|c|}{$2,841(30.8 \%)$} \\
\hline & Average ORF size (bp) & \multicolumn{2}{|c|}{996} & \multicolumn{2}{|c|}{990} & \multicolumn{2}{|c|}{989} \\
\hline \multirow[t]{2}{*}{ Core/quasi-core vs Noncore } & & 955 & 1,089 & 966 & 1,027 & 965 & 1,027 \\
\hline & Average Intergenic region size (bp) & \multicolumn{2}{|c|}{105} & \multicolumn{2}{|c|}{119} & \multicolumn{2}{|c|}{121} \\
\hline \multirow[t]{2}{*}{ Core/quasi-core vs Noncore } & & 101 & 116 & 115 & 126 & 118 & 126 \\
\hline & Coding density (\%) & \multicolumn{2}{|c|}{$90.4 \%$} & \multicolumn{2}{|c|}{$89.3 \%$} & & \\
\hline Core/quasi-core vs Noncore & & $90.5 \%$ & $90.3 \%$ & $89.4 \%$ & $89.1 \%$ & $89.0 \%$ & $89.1 \%$ \\
\hline RNA & rRNA operons & & & & & & \\
\hline Core/quasi-core vs Noncore & & 4 & 0 & 4 & 0 & 4 & 0 \\
\hline & tRNA genes & & & & & & \\
\hline Core/quasi-core vs Noncore & & 41 & 9 & 41 & 11 & 41 & 11 \\
\hline
\end{tabular}


Initiated from oriC, the $d n a A$ gene was chosen as the starting point for the numbering of the total CDSs in clockwise order (Figure 2). We assigned 5,518 CDSs (67.9\%) to known or putative functions, whereas the remaining 2,603 CDSs (32.1\%) were annotated as genes encoding hypothetical proteins (Table 1). The dissociated plasmid (designated as pXL100) encodes 49 genes, of which 43 are functionally unknown. Similar to $A$. mediterranei, the $A$. orientalis genome contains four rRNA operons (16S-23S$5 \mathrm{~S}$ ), and their 16S RNA sequences are at the range of $97 \%$ identical (Additional file 1: Table S1). Comparing the four rRNA operons within the $A$. orientalis genome, the first two, counted clockwise from $d n a A$, are both transcribed in the forward direction and their 16S rRNA sequences are slightly different (98-99\% identity). The second two are transcribed from the reverse strand and share identical sequences for their $16 \mathrm{~S}$ rRNAs (Additional file 1: Table S1). A. orientalis has 50 tRNA genes, which are largely similar to those of $A$. mediterranei, both in the chromosomal location and anticodon constitutions, with only a few exceptions, such as one less arginine and tyrosine tRNA genes and one more glutamic acid tRNA gene. It is worth emphasizing that, unlike A. mediterranei, no selenocysteine tRNA (tRNA ${ }^{\mathrm{Sec}}$ ) was found in the $A$. orientalis genome. Correspondingly, genes encoding selenocysteine synthase (selA), elongation factor (selB), and selenophosphate synthase (selD) were not found in the $A$. orientalis chromosome. Formate dehydrogenase, which has a selenocysteine (Sec)-encoding UGA codon found in the $A$. mediterranei genome, is also absent in A. orientalis. Compared with $A$. mediterranei, A. orientalis demonstrates a clearer sporulation phenotype (Figure 1). Although the genes responsible for this phenotypic difference are yet to be thoroughly defined, two genes, spsF (AORI_0253) and spsG (AORI_0254), encoding spore coat proteins, were identified only in the genome of $A$. orientalis. In contrast, the two pMEA100-like integrated plasmids found in the $A$. mediterranei genomes are absent from the genome of $A$. orientalis, whereas the free plasmid pXL100 present in A. orientalis $\mathrm{HCCB} 10007$ is not found in any other sequenced Amycolatopsis strains.

Reciprocal BLASTP was used to calculate the orthologs between $A$. orientalis and other related actinomycetes (A. mediterranei S699 and U32, Amycolatopsis sp. ATCC 39116, Saccharopolyspora erythraea, Streptomyces coelicolor, Saccharomonospora viridis, Nocardia farcinica, and Mycobacterium tuberculosis; Additional file 1: Figure S1). By employing a relatively strict condition (identity $>30 \%$, length coverage $>80 \%$ ), A. mediterranei (U32 or S699) shares $50.3 \%$ of the total CDSs (4,642 or $4,650)$ as orthologs with $A$. orientalis, which is the highest among all of the comparisons of the selected actinomycetes. The genome of Amycolatopsis sp. ATCC 39116 was recently sequenced by the DOE Joint Genome Institute
(JGI) [28], and a high-quality draft with 11 contigs was released in GenBank (accession no. AFWY00000000). We annotated it online using the fully automated service RAST [29] and found that, for the 8,328 predicted CDSs, Amycolatopsis sp. ATCC 39116 shares 4,165 orthologs (50.0\%) with $A$. orientalis (Additional file 1: Figure S1) S. erythraea shared 2,871 orthologs (39.9\%) with $A$. orientalis, which is the second highest number among sequenced actinomycetes other than that of Amycolatopsis, coincides with the close phylogenetic relationship between the two genera (Saccharopolyspora vs. Amycolatopsis). Although S. viridis shares only 2,318 orthologs with A. orientalis, with its small chromosome (4.3 Mbp) encoding 3,828 proteins, the genus Saccharomonospora represented by $S$. viridis is still considered phylogenetically close to Amycolatopsis, sharing an extremely high percentage (approximately 60.6\%) of orthologs, even higher than that between any two species of the same genus (Additional file 1: Figure S1).

\section{Genome configuration and plasticity of $A$. orientalis compared with $A$. mediterranei}

The unique chromosomal configuration consisting of core versus non-core regions characterized by the distinct features in gathering of essential genes (i.e., genes coding for functions of cell division, replication, transcription, translation, and amino-acid metabolism) in the corresponding genomic regions was first recognized in the linear genome of S. coelicolor [30] and then in the circular chromosome of S. erythraea [31]. Recently, a novel "quasi-core" region, with typical core characteristics, was defined within the non-core region of the A. mediterranei U32 genome, along with the proposition of three discriminable criteria, including the gathering of essential genes, the discrepancy in coding density of orthologous genes and the co-linearity of the orthologs' order [24]. In this study, taking the advantage of the availability of the complete genome sequences of two species (A. orientalis and A. mediterranei) from the same genus, the chromosomal configuration of these species was analyzed using more rigorous statistical methods, and special genomic plasticity related to major antibiotics production was revealed as probable chromosomal recombination events.

First, a core region of $A$. orientalis genome (nucleotide coordinates of 0-3.1 Mbp and 6.3-8.9 Mbp, corresponding to AORI_0001-AORI_2890 and AORI_5565-AORI_8121) was recognized by its good co-linearity of the order of its orthologs, with $14.5 \%$ of coding density for essential genes compared with $10.2 \%$ in non-core regions $(P<0.01)$. Meanwhile, the coding density of orthologous genes in the core region $(68.2 \%)$ was also higher than that in the noncore regions (37.3\%) $(P<0.01)$ (Figure 3A, Additional file 1: Table S2). 

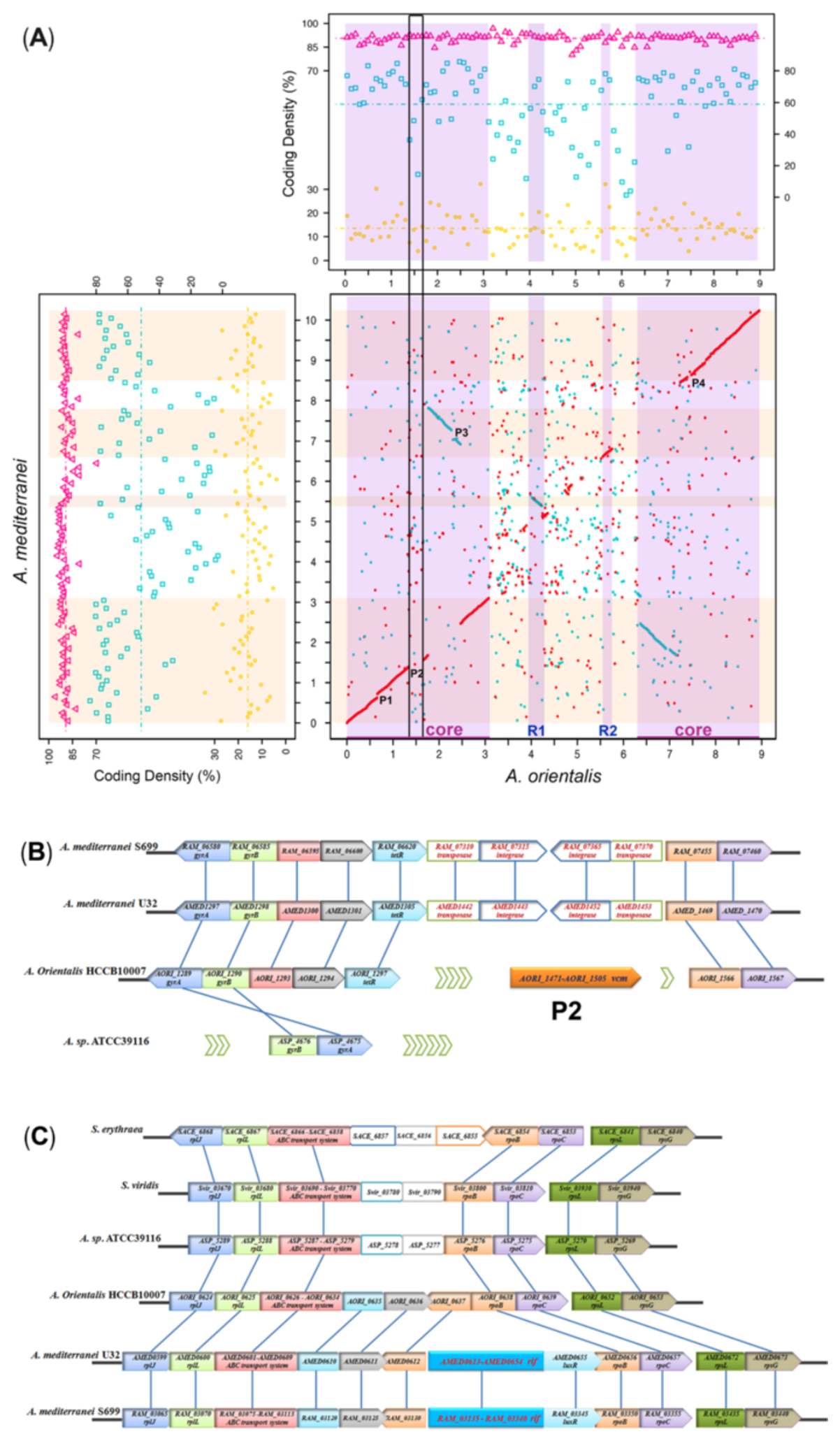

P1

Figure 3 (See legend on next page.) 
(See figure on previous page.)

Figure 3 Genome configurations of $\boldsymbol{A}$. orientalis and $\boldsymbol{A}$. mediterranei. (A) All of the dots in the panels were calculated in a $90-\mathrm{kb}$ sliding window. For the broken $X$ plot (lower right of the panel), the dots represent a reciprocal best match between the genomes of $A$. orientalis and A. mediterranei, based on the BLASTP comparison. The X-axis (Y-axis) is the nucleotide scale of the A. orientalis (A. mediterranei) chromosome. R1 (4.02-4.28 Mb, AORI_3663-AORI_3909) and R2 (5.55-5.75 Mbp, AORI_4997-AORI_5173) were designated as the two quasi-core regions in the A. orientalis genome. Reciprocally, two regions (AMED_4864-AMED_5049 and AMED_5970-AMED_7071) were defined as the quasi-core in the A. mediterranei genome. The core and quasi-core regions are highlighted in lavender (A. orientalis) or in pink (A. mediterranei). P1 to P4 were designated as the regions containing biosynthesis clusters of rifamycin (rif in A. mediterranei), vancomycin ( $v c m$ in A. orientalis), NRPS ( $n r p s$ iO in A. mediterranei) and polyketide (pks9 in A. orientalis), respectively. In the upper right and lower left panels, the pink triangles represent the coding density of all of the genes; the turquoise squares represent the coding density of orthologs between the genomes of $A$. orientalis and $A$. mediterranei; and the yellow circles represent the coding density of the essential genes. The area within the black square frame is the P2 region containing the $v \mathrm{~cm}$ cluster, with a lower coding density of orthologs and essential genes. (B) Alignment of the P2 region with its flanking genes related to the vancomycin biosynthesis in selected actinomycete genomes. The green arrows represent the omitted genes in the corresponding genomes. (C) Alignment of the P1 region with its flanking genes related to the rifamycin biosynthesis in selected actinomycete genomes. All of the genome data are available at NCBI.

Second, different from the analysis between the genomes of $A$. mediterranei and S. erythraea, two quasicore regions ( $\mathrm{R} 1$ and $\mathrm{R} 2$ ) within the non-core of the $A$. orientalis genomes are defined compared with that of $A$. mediterranei (Figure 3A). The gene orders in these two regions show good conservation with those of $A$. mediterranei and the coding density of orthologous genes is 67.0\% (R1, $P<0.05)$ and 73.3\% (R2, $P<0.01)$, respectively, significantly higher than that of the non-core regions (Additional file 1: Table S2). In addition, the coding density of essential genes in these two regions is also higher than that in the non-core regions (10.2\%), reaching $16.0 \%(\mathrm{R} 1, P \approx 0.05)$ and $22.3 \%(\mathrm{R} 2, P<0.05)$, respectively (employing $45-\mathrm{kb}$ instead of $90-\mathrm{kb}$ sliding window size for statistical analysis, as shown in Additional file 1: Table S2). It is worth mentioning that the identification of two quasi-cores is reciprocal, i.e., two regions (AMED_4864-AMED_5049 and AMED_5970AMED_7071) can be defined as the quasi-cores in the $A$. mediterranei genome by comparison with the genome of $A$. orientalis (Figure 3A), which was obviously unrecognized previously when the genomes of differently related species were compared [24]. In particular, we noticed that all of the four rRNA operons of both species are located in either the core or quasi-core regions (Additional file 1: Table S1 and Figure 2), as are 41 of the 50 tRNA genes of $A$. orientalis (52 of A. mediterranei) containing the codons for all 20 essential amino acids (Table 1).

Comparing the genome of $A$. orientalis with that of $A$. mediterranei, a large inversion usually known as the " $\mathrm{X}$ pattern" was revealed. Although the order of orthologs is well conserved between these two species, the line of the "X pattern" is not consecutive and is often interspersed with break points. Most of the break points are within the non-core regions encoding the majority of the secondary metabolite biosynthesis gene clusters (Figure 2 and Figure 3A), which might represent some horizontal gene transfer events. The rare break points embedded in the core regions, termed P1 to P4, are usually the regions containing gene clusters for the synthesis of the "speciesspecific" secondary metabolites, i.e., rifamycin (rif) in $\mathrm{P} 1$ of $A$. mediterranei and vancomycin $(\nu \mathrm{cm})$ in $\mathrm{P} 2$ of $A$. orientalis (Figure $3 \mathrm{~A})$. The $\mathrm{P} 2$ region in A. orientalis is nearly $300 \mathrm{~kb}$ in length. It not only contains the $64 \mathrm{~kb}$ $v \mathrm{~cm}$ cluster, but also encodes many hypothetical proteins or predicted transcriptional regulators, and thus shows a relatively low coding density of orthologs and essential genes. In contrast, the corresponding region of A. mediterranei contains dozens of CDSs (over less than $100 \mathrm{~kb}$ ), including two gene pairs of transposase/integrase (AMED_1442-AMED_1443 and AMED_1452-AMED_1453; Figure 3B). The AMED_1452-AMED_1453 gene pair is a duplicate of AMED_1442-AMED_1443 with a reversed transcription direction, which indicates that an insertion might have occurred in the P2 region of an ancestral strain, which resulted in the acquisition of the $v \mathrm{~cm}$ gene cluster in A. orientalis. Unlike the P2 flanking regions, the two regions flanking the rif cluster of $\mathrm{P} 1$ are highly conserved among the Amycolatopsis species (Figure 3C). As indicated by the alignments, the rif cluster appears to be inserted between two genes encoding a conserved hypothetical protein (AMED_0612) and the unique DNAdirected RNA polymerase $\beta$ subunit (RpoB, AMED_0656). Therefore, we speculate that the ancestor of $A$. mediterranei may have acquired the rif cluster more recently than that occurred in P2. In addition, a LuxR-like transcriptional regulator (AMED_0655) is located between the 3 ' end of the rif cluster and the conserved rpoB gene (Figure $3 \mathrm{C}$ ), which seems to have been acquired simultaneously with the rif cluster by the ancestral strain. A potential regulatory function of this LuxR-like protein in rifamycin biosynthesis is inferred, and the corresponding experimental proof is currently being pursued (unpublished data).

In this study, intra-generic comparative genomic analysis of Amycolatopsis not only confirmed the core/quasi-core and non-core genomic configuration, but also discovered 
certain genomic plasticity hot spots in this genus. It should be noted that the definition of core, quasi-core, or non-core regions of a genome so far remains a relative concept with respect to the genomes of certain species or genera to be compared. The choice of the sliding window size could also influence the characterization of the genomic configuration, which was clearly demonstrated in the case of comparing the coding density of essential genes between the quasi-core and non-core regions. When the window size used in the analysis was reduced from $90-\mathrm{kb}$ to $45-\mathrm{kb}$, thus doubling the sample size, the $P$-values were reduced from more than one to $\leq 0.05$ (Additional file 1: Table S2). In our opinion, these flexible categorizations are somewhat artificial; however, they are useful tools to infer different processes of evolution of a genus or of microevolution of a species. The quasi-core region(s) may represent the residue(s) of the complex evolution dynamics (vertical genomic recombination events) of the ancestral genome, while the non-core regions may represent the chromosomal expansions (horizontal gene transfer) in the various descendants' genomes. As more whole genome sequences of different strains of one species or different species of one genus, as well as those from closely related genera, are published, the biological implications of this genomic plasticity in bacterial phylogeny will be clarified.

\section{Development of molecular chemotaxonomic characteristics (MCCs) for the genus of Amycolatopsis}

The taxonomic status of $A$. orientalis underwent the same revision history as that of $A$. mediterranei [24]; i.e., it was originally considered a streptomycete [32], then transferred to Nocardia [33], and finally classified as a species of the newly established genus Amycolatopsis [34], which was typically defined by the biochemical characteristics of its cell wall (chemotype IV) and cell membrane (chemotype II). As initiated in the study of the A. mediterranei U32 genome [24], in addition to the molecular genetic basis responsible for the components of arabinose, glycine, diaminopimelic acids and mycolic acids (Additional file 1: Table S3, Additional file 1: Table S4, Additional file 1: Figure S2, and Additional file 1: Figure S3), we attempted to analyze the previously unidentified genetic basis of two more chemotaxonomic phenotypes, i.e., phospholipids and menaquinones.

The cell membrane of actinomycetes is classified into five types according to the presence of certain nitrogenous phospholipids [35]. The Amycolatopsis cell membrane belongs to the PII type because only one nitrogenous phospholipid, namely phosphatidyl ethanolamine (PE), was usually detected in its cell membrane [35]. In prokaryotes, phosphatidylserine is first generated from CDP-diacylglycerol, a general intermediate for the synthesis of different types of phospholipids, catalyzed by phosphatidylserine synthase (PssA, EC: 2.7.8.8), and is then transformed into $\mathrm{PE}[36,37]$ by phosphatidylserine decarboxylase (Psd, EC: 4.1.1.65) (Figure 4A). Orthologs of both pss A (AORI_7346) and psd (AORI_7345) could be identified in the $A$. orientalis genome. These two genes also exist in other actinomycetes with a type PII cell membrane, such as A. mediterranei, N. farcinica, S. coelicolor, and $M$. smegmatis, but are absent in actinomycetes with a type PI membrane (no nitrogenous phospholipids) or other types of cell membranes. Moreover, in the genomes of neither $A$. orientalis nor A. mediterranei did we identify genes encoding phosphatidylcholine synthase (Pcs, EC: 2.7.8.24), which catalyzes the formation of phosphatidyl choline (PC), the characteristic type PIII phospholipid, or the genes encoding phosphatidylglycerophosphatase A (PgpA, EC: 3.1.3.27), which catalyzes the formation of phosphatidyl glycerol (PG), the characteristic type PV phospholipid (Figure 4A). It is worth mentioning that the gene ept1, which encodes ethanolamine phosphotransferase (EPT1, EC: 2.7.8.1) that catalyzes the biosynthesis of PE from 1, 2-diacylglycerol and CDPethanolamine in eukaryotes, is also absent in any of the sequenced actinomycete genomes (Figure 4A).

Isoprenoid quinones comprise a hydrophilic head and an apolar isoprenoid side chain, functioning mainly as electron and proton carriers in photosynthetic and respiratory electron transport systems [38]. These compounds have also been used as conventional biomarkers in bacterial chemotaxonomy since the 1960s $[39,40]$. In the synthesis of isoprenoid quinones, isoprenyl diphosphate synthase (Isp) catalyzes the consecutive condensation of isopentenyl diphosphate (IPP) with allylic diphosphates and produces a variety of prenyl diphosphates with different chain lengths [38]. Previous studies reported that the specific amino-acid residues of isoprenyl diphosphate synthases attributable to the chain-length determination were designated the chain-length determination (CLD) region [41]. However, for the biosynthesis of isoprenoid quinones with longer chain lengths (more than C30), the consensus CLD region in isoprenyl diphosphate synthases has yet to be clarified [41]. In actinomycetes, menaquinone (MK) is the characteristic type of isoprenoid quinone in the cell membrane. We compared the amino-acid sequences (particularly the CLD region) of Isp from type strains of actinomycetes harboring different-length MKs but no regular patterns could be found (Figure 4B). Hence, the isoprenyl diphosphate synthases were analyzed phylogenetically using the neighbor joining (NJ) method. As shown in Figure 4B, species with MK-7 (C35) in their membranes are clustered within one clade, whereas species with MK-8 (C40) or MK-9 (C45) are clustered together and are indistinguishable in the tree. In addition, the maximum parsimony (MP) method was also used to construct a phylogenetic tree. However, the clustering result could not distinguish the 


\section{A}

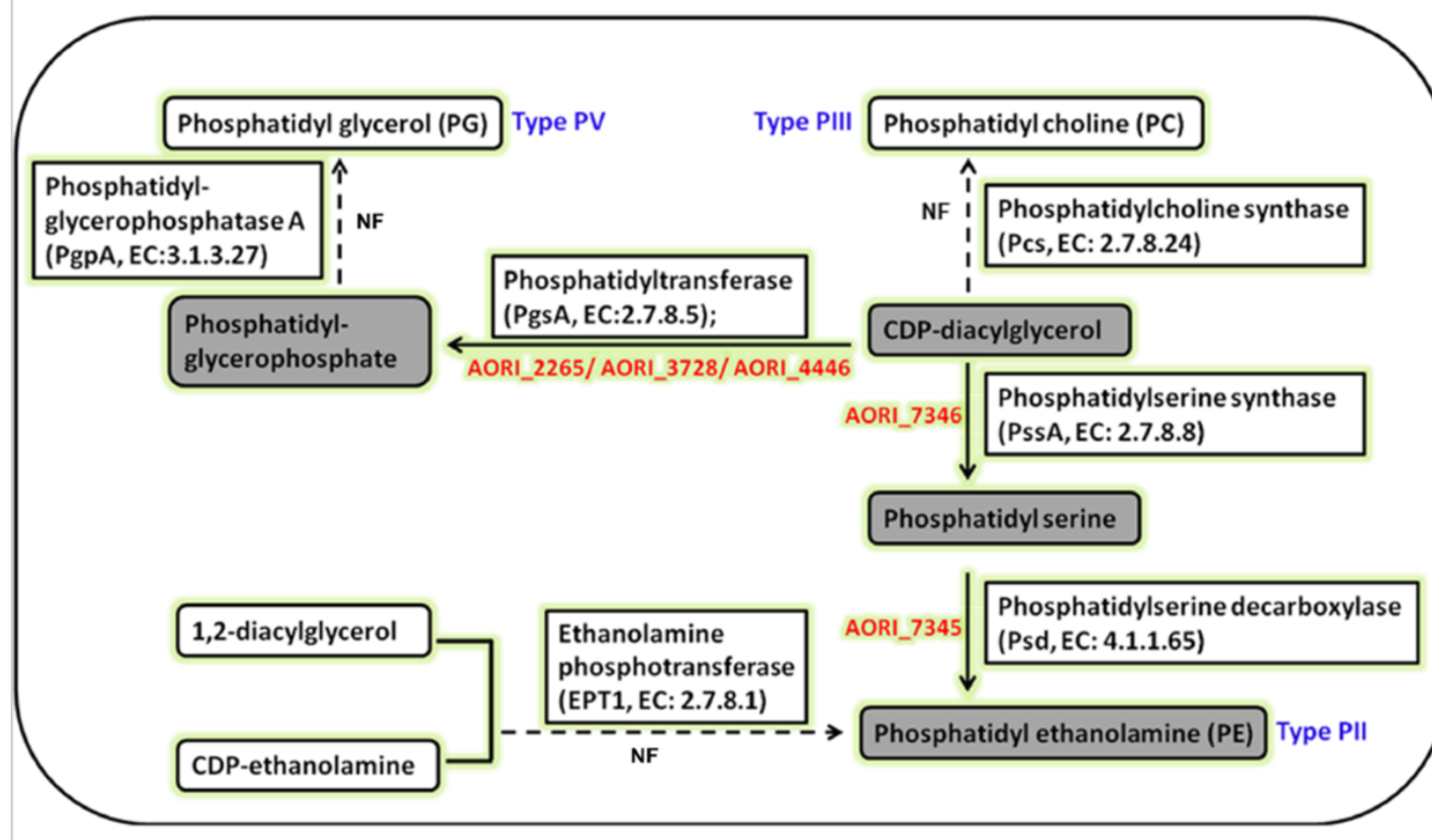

B

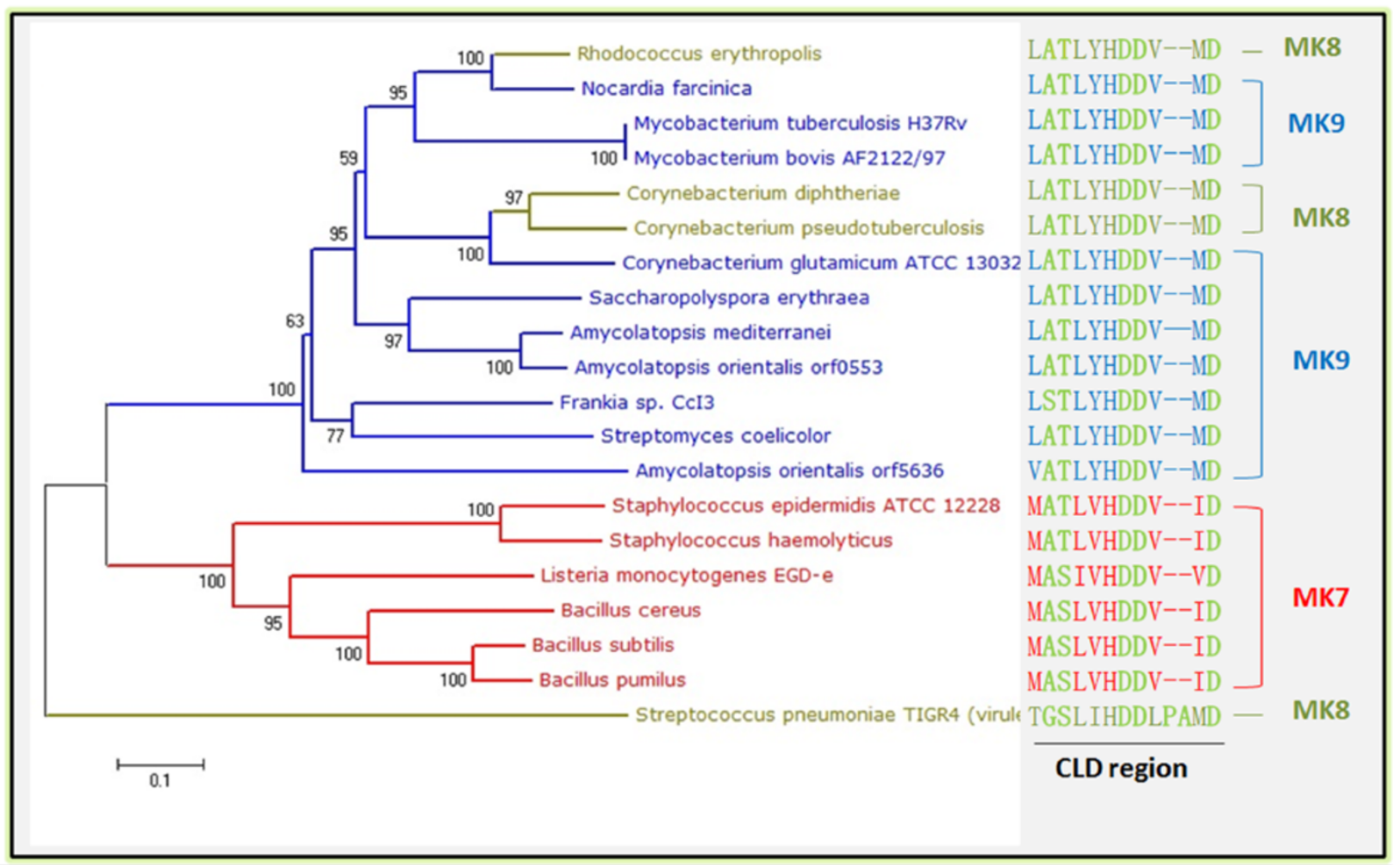

Figure 4 (See legend on next page.) 
(See figure on previous page.)

Figure 4 Biosynthetic pathways of different types of nitrogenous phospholipids in actinomycetes. (A) The cell membrane of Amycolatopsis belongs to the type PII because PE is the dominant phospholipid detected. Two essential proteins (AORI_7345 and AORI_7346, labeled in red color) involved in the biosynthesis of PE were encoded by the A. orientalis genome, whereas the genes encoding enzymes involved in other types of nitrogenous phospholipids were not found (NF). Actinomycetes of type PI contain no nitrogenous phospholipids in their cell membrane, while type PII, type PIII, type PIV, and type PV actinomycetes contain the following characteristic phospholipids: PE, PC, GluNU, and PG, respectively. Panel (B) illustrates the analysis of isoprenyl diphosphate synthases from type strains of actinomycetes. The names and amino-acid sequences of the strains with different colors represent actinomycetes harboring different-length MKs: red, MK7 (C35); olive-green, MK8 (C40); blue, MK9 (C45). The amino-acid sequences of the chain-length determination (CLD) region are emphasized in green on the right of the panel. The protein sequences were obtained from NCBI at http://www.ncbi.nlm.nih.gov/protein/.

species harboring MK-8 or MK-9 either (Additional file 1: Figure S4). Therefore, it is yet to be experimentally clarified whether the genotypes of isoprenyl diphosphate synthases, i.e., their sequence specificities, are a sufficient determinant for all different side chain lengths of isoprenoid quinone, or whether the variation of isoprenoid quinones in actinomycetes is a quantitative, rather than a qualitative, property that might be determined by gene expression regulation or other post transcription/translational modifications.

In summary, our sequential studies in two species of Amycolatopsis (ref to [24] and this work) indicate that the chemotaxonomic characteristics of this genus, which relate to, but differentiate from Streptomyces and Nocardia, are intrinsically determined by the molecular phylogeny of their encoding genes. On the other hand, the failure to precisely determine the molecular genetic mechanisms underlying the chain-length of MK hinted at the complexity of these genotype/phenotype correlations. Together with some more important chemotaxonomic characteristics, such as the composition of fatty acids, these complex phenotypes and their related molecular genetic mechanisms may prompt further biochemical and molecular biological studies. Nowadays, we propose that, based on whole genome analysis of multiple bacterial strains belonging to and related with a taxon (particularly, species or genus), potential molecular chemotaxonomic characteristics (MCCs) could be developed as the genotypes underlie the biochemical characteristics (phenotypes) of the taxon. The implementation of MCCs in bacterial systematics will not only alleviate the tedious workload of chemotaxonomic identification, but also improve our understanding of the genetics of bacterial metabolomes, which will form an indispensable portion of the modern prokaryotic taxonomy in the era of genomics [42].

\section{Biosynthesis of secondary metabolites and the post-assembly modifications of vancomycin in $A$. orientalis}

Twenty-six secondary metabolite biosynthetic gene clusters were predicted in the complete genome of $A$. orientalis HCCB10007, including nine type I polyketide synthase (PKS) clusters, one type II PKS cluster, ten non-ribosomal peptide synthetase (NRPS) clusters, three hybrid PKS-
NRPS clusters, two clusters for terpenoids, one cluster for lycopene $(l y c)$, and one cluster for $\beta$-carotene (car) (Figure 2). The total length of these gene clusters was estimated $\sim 552 \mathrm{~kb}$, which is $6.2 \%$ of the whole genome. In contrast to the essential genes, most of the secondary metabolite biosynthetic gene clusters (18 out of 26) were in the non-core regions (Figure 2).

To determine the possible phylogenetic relationships of the secondary metabolites biosynthesis gene clusters, all of the CDSs for PKSs, NRPSs, or terpene synthases were compared against the NCBI database via BLASTP. The best hits information is provided in Additional file 1: Table S5. Twenty-seven genes in nine biosynthetic gene clusters (34.6\% of the total 26 clusters) have orthologs in the $A$. mediterranei genome with the best hitting scores, i.e., car, $p k s 1, l y c$, and tps 2 in the core region and $p k s 3, t p s 1, n r p s 7, p k s 5$, and $p k s 6$ in the non-core regions. Furthermore, the $n r p s 7, p k s 5$, and $p k s 6$ gene clusters are closely located in the non-core region, particularly the $p k s 5$ and $p k s 6$ clusters (Figure 2). These close correlations between sequence similarity and genomic loci gathering may indicate a common phylogenetic origin.

Notably, among the eight gene clusters for secondary metabolism located in the core and quasi-core regions, except for four clusters (car, pks1, lyc, and tps2) orthologous to those encoded in $A$. mediterranei genome, all of the other $A$. orientalis specific clusters ( $v c m$, pks9, nrps10, nrps4) are located in the break point of the chromosomal "X pattern" blocks. However, because of the small coding size of nrps10 and nrps4, only the $v \mathrm{~cm}$ cluster $(64 \mathrm{~kb})$, located in the P2 break point, and the pks9 cluster (AORI_6587-6642, $61.7 \mathrm{~kb}$ ), located in the P4 break point, could be traced in the "X pattern" blocks (Figure 3A). The KS domains of $p k s 9$ are similar to those of the salinosporamide A biosynthetic gene cluster in Salinispora tropica CNB-440 (73\% identity) [43]. This cluster is rich in genes encoding modification enzymes, such as glycosyltransferases, halogenase, and cytochrome P450, which suggests the production of a glycosidic and halogenic compound.

In the non-core regions, cluster pks2 (AORI_2937-2956, $79.6 \mathrm{~kb}$ ) contains a type I polyketide synthase, which was once reported to synthesize a glycosidic polyketide ECO- 
0501 that shows activities against MRSA and VRE [44]. For the other secondary metabolite biosynthesis gene clusters in A. orientalis genome, their putative substrates or probable products were predicted using catalytic domain analysis against the SBSPKS [45] or NRPSDB [46] databases and the results are listed in Additional file 1: Table S5. We isolated the total RNA of A. orientalis from two different cultures (fermentation medium F1 and nutrient medium F5, Additional file 1: Supplementary Materials and Methods), and used reverse-transcription PCR to detect the transcription profiles of the gene clusters that might synthesize potential secondary metabolites. As shown in the Additional file 1: Figure S5, in both F1 and F5 media, the genes of three clusters ( $p k s 5, n \_p 2$, and $v \mathrm{~cm}$ ) showed significant levels of transcription, with $v \mathrm{~cm}$ being the highest among all gene clusters tested. Another cluster (nrps4) was expressed in the F1 fermentation medium but not in the F5 medium. Although we failed to identify any novel secondary metabolites, our data provides a foundation for further exploration.

The $v \mathrm{~cm}$ cluster was annotated to encode a total of 35 enzymes (AORI_1471 to AORI_1505), including three vancomycin-resistance proteins (VanH, VanA, and VanX $[7,8])$, three large NRPSs, several post-assembly tailoring enzymes, and a series of biosynthetic proteins for the supply of amino-acid precursors (Table 2). Different from the cep and bal clusters, in which three genes encoding glycosyltransferases were predicted [7,8], only two glycosyltransferases are encoded in the $v \mathrm{~cm}$ cluster (AORI_1486 and AORI_1487). On the other hand, the vancomycinresistance genes vanHAX (AORI_1471-AORI_1473) are only predicted in $v \mathrm{~cm}$ and not in the other two clusters (Table 2). Throughout the $A$. orientalis genome, we identified another vanA (AORI_8112) and vanX (AORI_2227), as well as a two-component system (AORI_7254-AORI_ 7255) similar to the vanSR of bal that may be related to the vancomycin resistance.

Similar to the biosynthesis of balhimycin and chloroeremomycin, the biosynthesis of vancomycin includes three steps [17]. The related functional genes inside and outside of the $v \mathrm{~cm}$ cluster were mapped to the A. orientalis genome (Figure 5). First, seven amino-acid precursors, including one leucine, one asparagine, two $\beta$-hydroxytyrosine $(\mathrm{L}-\beta \mathrm{Ht})$, two 4-hydroxyphenylglycine (L-Hpg) and one 3, 5-dihydroxyphenylglycine (L-Dpg), need to be synthesized. Genes encoding the enzymes responsible for the biosynthesis of three non-protein amino acids were identified in the genome, i.e., AORI_1492-AORI_1494 for L$\beta \mathrm{Ht}$, AORI_1476, AORI_1491, AORI_1495-AORI_1496 for L-Hpg, and AORI_1502-AORI_1505 for L-Dpg.

Second, the seven precursor amino acids are assembled to form a heptapeptide backbone, which are catalyzed by the NRPSs VcmA (AORI_1478), VcmB (AORI_1479), and VcmC (AORI_1480). These three giant enzymes contain seven modules (M1-M7) with 24 domains that function in the selection, activation, condensation and epimerization of the amino-acid substrates [17]. In M2, M4, and M5, there are three epimerases (E domain) that convert L-

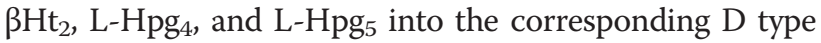
amino acids. The N-terminal amino acid of vancomycin is D-methylleucine [47]. However, neither an epimerase nor a dual condensation/epimerization domain $[48,49]$ was observed in M1 or the adjacent C domain in M2. Rausch et al. conjectured that a racemase outside the $v \mathrm{~cm}$ cluster might be responsible for the conversion of L-leucine into D-leucine, which can be incorporated directly into the glycopeptides [48]. Throughout the whole genome, there are 11 genes that potentially encode racemases, including six amino-acid racemases, three CoA racemases, and two mandelate racemases (see Additional file 1: Table S6). The recent genomic analysis of Vibrio cholera identified a novel PLP-dependent amino-acid racemase (vc1312) that was proven to be necessary and sufficient for the synthesis of the unusual D-amino acids, including D-leucine [50]. With vc1312 as the query sequence, we used the BLASTP program to search throughout the whole genome of A. orientalis. The results revealed one protein, annotated as an amino-acid racemase (AORI_0725), which has 28\% amino-acid identity (48\% positive) with vc1312 and that may function in D-leucine conversion. Further experimental proof is required to confirm its involvement in vancomycin synthesis.

The last step is the post-assembly modifications of the heptapeptide backbone, including its cyclization, halogenation, methylation and glycosylation. Based on their corresponding genes in the cep and bal clusters [7,8], the functions of the modification genes in the $v \mathrm{~cm}$ cluster were annotated (Table 2). The oxyA/B/C (AORI_14821484) genes likely encode three $\mathrm{P} 450$ monooxygenases that are responsible for closing the linear peptide to form the heptapeptide ring [19,51]. Adjacent to them, AORI_1485 ( vhal) is predicted to encode a halogenase, showing $94 \%$ amino-acid sequence identity with that encoded by the bhaA in bal [9], which chlorinates the $\beta \mathrm{Ht}$ residues. However, the exact timing of the chlorination is unknown, although it was proposed to occur before the oxidative couplings [52]. The methylation of D-leucine on the $\alpha-\mathrm{NH}_{2}$ is catalyzed by a methyltransferase, which has been functionally characterized in the cep cluster [14]. Its orthologous protein in the $v \mathrm{~cm}$ cluster was found and annotated as Vmt (AORI_1490). Glycosylation is the last of the modifications and the functional glycosyltransferases for vancomycin biosynthesis have been well-characterized biochemically [10,15]. GtfE (AORI_1487) is responsible for the addition of the first TDP-glucose moiety to the 4'-hydroxyl group of amino acid $\mathrm{Hpg}_{4}$, and the other glycosyltransferase GtfD (AORI_1486) adds the second TDP-L- $\beta$-vancosamine moiety to the 2 '-hydroxyl group of 
Table 2 Annotation of the $\mathbf{v c m}$ cluster in A. orientalis and the comparison with bal and cep

\begin{tabular}{|c|c|c|c|c|}
\hline AORI_CDS & Gene name & Annotation & Best hit genes (NCBI accession no.) & Identity \% \\
\hline AORI_1471 & vanH & D-lactate dehydrogenase & - & - \\
\hline AORI_1472 & vanA & D-alanine-D-alanine ligase & - & - \\
\hline AORI_1473 & $\operatorname{van} X$ & D-alanyl-D-alanine dipeptidase & - & - \\
\hline AORI_1474 & & Hypothetical protein & - & - \\
\hline AORI_1475 & ver & Regulator protein & IcI|Y16952.3_cdsid_CAG25754.1 & 83.33 \\
\hline AORI_1476 & $p d h$ & Prephenate dehydrogenase & IcI|Y16952.3_cdsid_CAG25755.1 & 84.12 \\
\hline AORI_1477 & & ATP-binding cassette, subfamily B & IC||AJ223999.1_cdsid_CAA11793.1 & 88.89 \\
\hline AORI_1478 & $v c m A$ & Non-ribosomal peptide synthetase & IC||AL078635.1_cdsid_CAB45052.1 & 81.31 \\
\hline AORI_1479 & $v c m B$ & Non-ribosomal peptide synthetase & IC||AJ223999.1_cdsid_CAA11795.1 & 82.34 \\
\hline AORI_1480 & vemC & Non-ribosomal peptide synthetase & |c||Y16952.3_cdsid_CAC48362.1 & 86.81 \\
\hline AORI_1481 & $\mathrm{mbtH}$ & MbtH protein & Ic||Y16952.3_cdsid_CAC48363.1 & 89.86 \\
\hline AORI_1482 & oxyA & Cytochrome P450 & Ic||Y16952.3_cdsid_CAA76547.1 & 86.19 \\
\hline AORI_1483 & $O x y B$ & Cytochrome P450 & Icl|Y16952.3_cdsid_CAA76548.1 & 87.44 \\
\hline AORI_1484 & oxyC & Cytochrome P450 & Ic||AJ223998.1_cdsid_CAA11791.1 & 91.69 \\
\hline AORI_1485 & vhal & Halogenase & Icl|Y16952.3_cdsid_CAA76550.1 & 93.89 \\
\hline AORI_1486 & $g t f D$ & Vancosaminyl transferase & Ic||Y16952.3_cdsid_CAA76553.1 & 69.93 \\
\hline AORI_1487 & gtfE & Glycosyl transferase & Icl|Y16952.3_cdsid_CAA76552.1 & 81.17 \\
\hline AORI_1488 & vcaC & Methyltransferase family protein & |c||Y16952.3_cdsid_CAC48364.1 & 93.87 \\
\hline AORI_1489 & & LmbE family protein & Ic||Y16952.3_cdsid_CAC48365.1 & 75.91 \\
\hline AORI_1490 & $v m t$ & Methyltransferase & IC||AJ223998.1_cdsid_CAA11779.1 & 73.21 \\
\hline AORI_1491 & hpgT & 4-hydroxyphenylglycine aminotransferase & IC||AJ223998.1_cdsid_CAA11790.1 & 89.7 \\
\hline AORI_1492 & $v h p$ & Hydrolase & IC||AJ223998.1_cdsid_CAA11784.1 & 85.87 \\
\hline AORI_1493 & $v c m D$ & Non-ribosomal peptide synthetase & IC||AJ223998.1_cdsid_CAA11773.1 & 83.57 \\
\hline AORI_1494 & $\operatorname{oxy} D$ & Cytochrome P450 & Ic||Y16952.3_cdsid_CAC48370.1 & 85.35 \\
\hline AORI_1495 & hmas & 4-hydroxymandelate synthase & IC||AJ223998.1_cdsid_CAA11761.1 & 75.56 \\
\hline AORI_1496 & hmo & 4-hydroxymandelate oxidase & |cl|Y16952.3_cdsid_CAC48372.1 & 85.24 \\
\hline AORI_1497 & & Antipoter & Ic||Y16952.3_cdsid_CAC48373.1 & 80 \\
\hline AORI_1498 & $v c a A$ & Oxidase & ICl|Y16952.3_cdsid_CAC48374.1 & 88.04 \\
\hline AORI_1499 & vcaE & Reductase & ICI|Y16952.3_cdsid_CAC48375.1 & 84.21 \\
\hline AORI_1500 & $v c a B$ & Aminotransferase & IC||AJ223998.1_cdsid_CAA11782.1 & 88.62 \\
\hline AORI_1501 & $v c a D$ & Epimerase & |cl|Y16952.3_cdsid_CAC48377.1 & 85.57 \\
\hline AORI_1502 & $d p g A$ & Polyketide synthase & IC||AJ223998.1_cdsid_CAA11765.1 & 92.9 \\
\hline AORI_1503 & $d p g B$ & Isomerase & |c||Y16952.3_cdsid_CAC48379.1 & 75 \\
\hline AORI_1504 & $d p g C$ & Thioesterase & |cl|Y16952.3_cdsid_CAC48380.1 & 83.25 \\
\hline AORI_1505 & $d p g D$ & Dehydration protein & Ic||Y16952.3_cdsid_CAC48381.1 & 86.49 \\
\hline
\end{tabular}

Note: "-" represents no orthologous gene was found in bal (Y16952.3) or cep (AL078635.1, AJ223999.1 and AJ223998.1) clusters.

a glucose residue. AORI_1487 shows the highest amino acid sequence similarity to BgtfB encoded by bal (81\%) and GtfB encoded by cep (81\%), whereas AORI_1486 shows the highest similarity to BgtfC encoded by bal (70\%) and GtfC encoded by cep (69\%). No glycosyltransferase corresponding to BgtfA or GtfA, which add 4-epivancosamine to the amino-acid residue of $\beta \mathrm{Ht}_{6}$ in bal or cep, was found in the genome of $A$. orientalis. Therefore, there is no epivancosamine moiety present in vancomycin (Figure 1).
To characterize the in vivo functions of the predicted halogenase, the putative methyltransferase and the glycosyltransferases encoded by the $v \mathrm{~cm}$ cluster, in-frame monogenic mutants of AORI_1485 (vhal), AORI_1490 (vmt), AORI_1486 (gtfD), and AORI_1487 (gtfE) are successfully constructed using a homologous recombination method similar to the PCR-targeting system (Methods). Various types of vancomycin derivatives, i.e., dechlorovancomycin, demethylvancomycin, desvancosamine vancomycin, and 


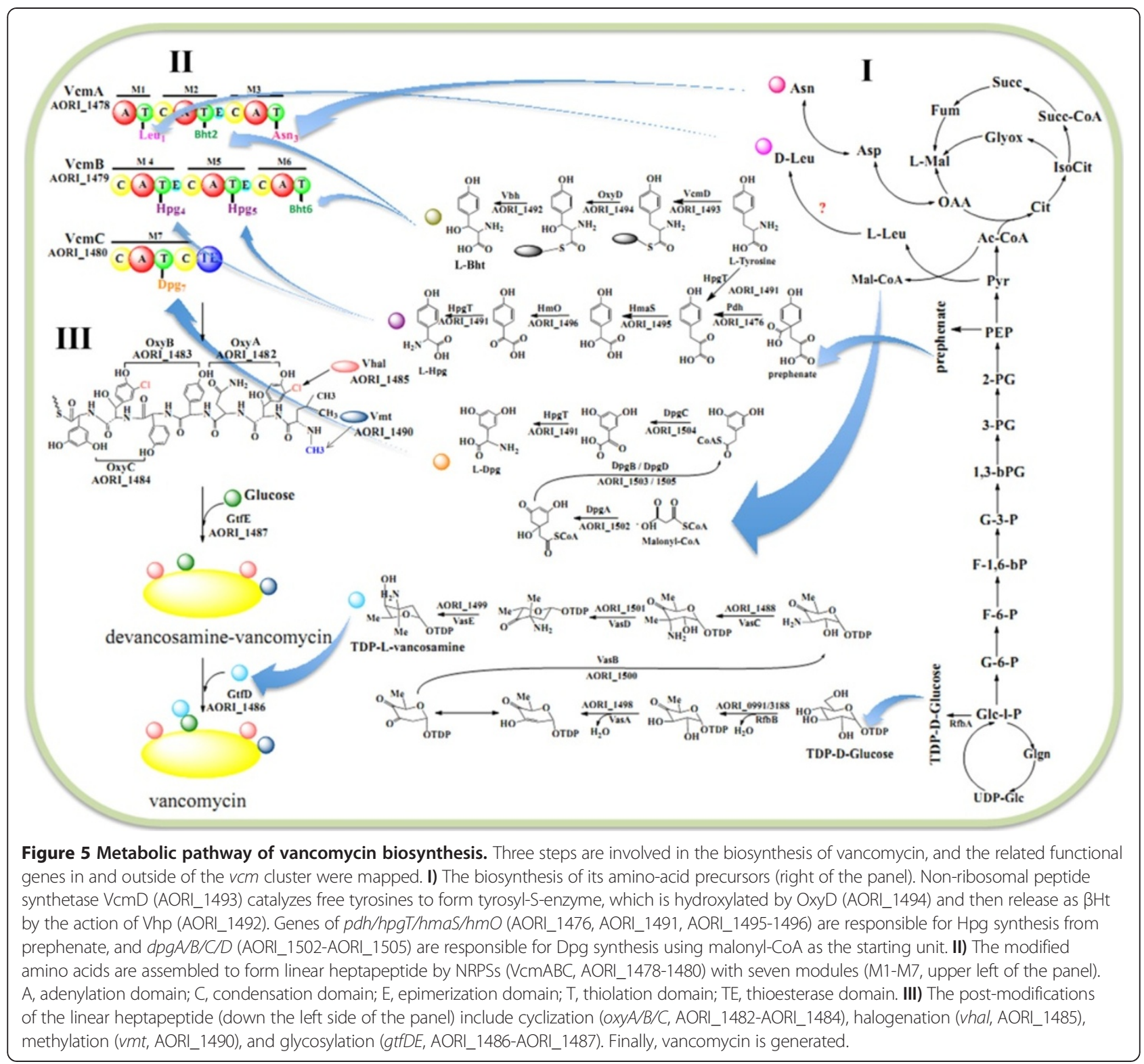

aglucovancomycin, which accumulated in the corresponding mutant cultures, were collected and their structures were confirmed by high-performance liquid chromatographymass spectrometry (HPLC-MS) (Figure 6). Based on the results from the zone of inhibition test, desvancosamine vancomycin (Figure 6C), particularly dechlorovancomycin (Figure 6B), showed a lower bioactivity relative to that of vancomycin, whereas aglucovancomycin (Figure 6D) showed a slightly higher bioactivity than that of vancomycin. The bioactivity of demethylvancomycin (Figure 6F) was comparable to that of vancomycin. In addition, using demethylvancomycin or aglucovancomycin as the substrate, dimethylvancomycin (Figure 6G) or dimethylaglucovancomycin (Figure 6E) were generated in vitro catalyzed by the heterogeneously expressed methyltransferase
AORI_1490. Their molecular weights were also confirmed by the HPLC-MS spectrum, and the positions of the two methyl groups on the $\mathrm{N}$ terminus of leucine (Figure 1) were further examined using nuclear magnetic resonance (NMR) (Additional file 1: Table S7, Additional file 1: Table S8). Compared with that of vancomycin (Figure 6A), dimethylvancomycin showed a comparable antibacterial activity. Although dimethylaglucovancomycin (Figure 6E) is a novel compound, its activity was also similar to that of aglucovancomycin. Taken together, both methylation and demethylation do not affect the in vitro antibacterial activity of vancomycin or its derivatives. For glycosylation, despite aglucovancomycin showing a slightly higher bioactivity than that of vancomycin in vitro, the in vivo activity was five-fold lower than that of vancomycin 


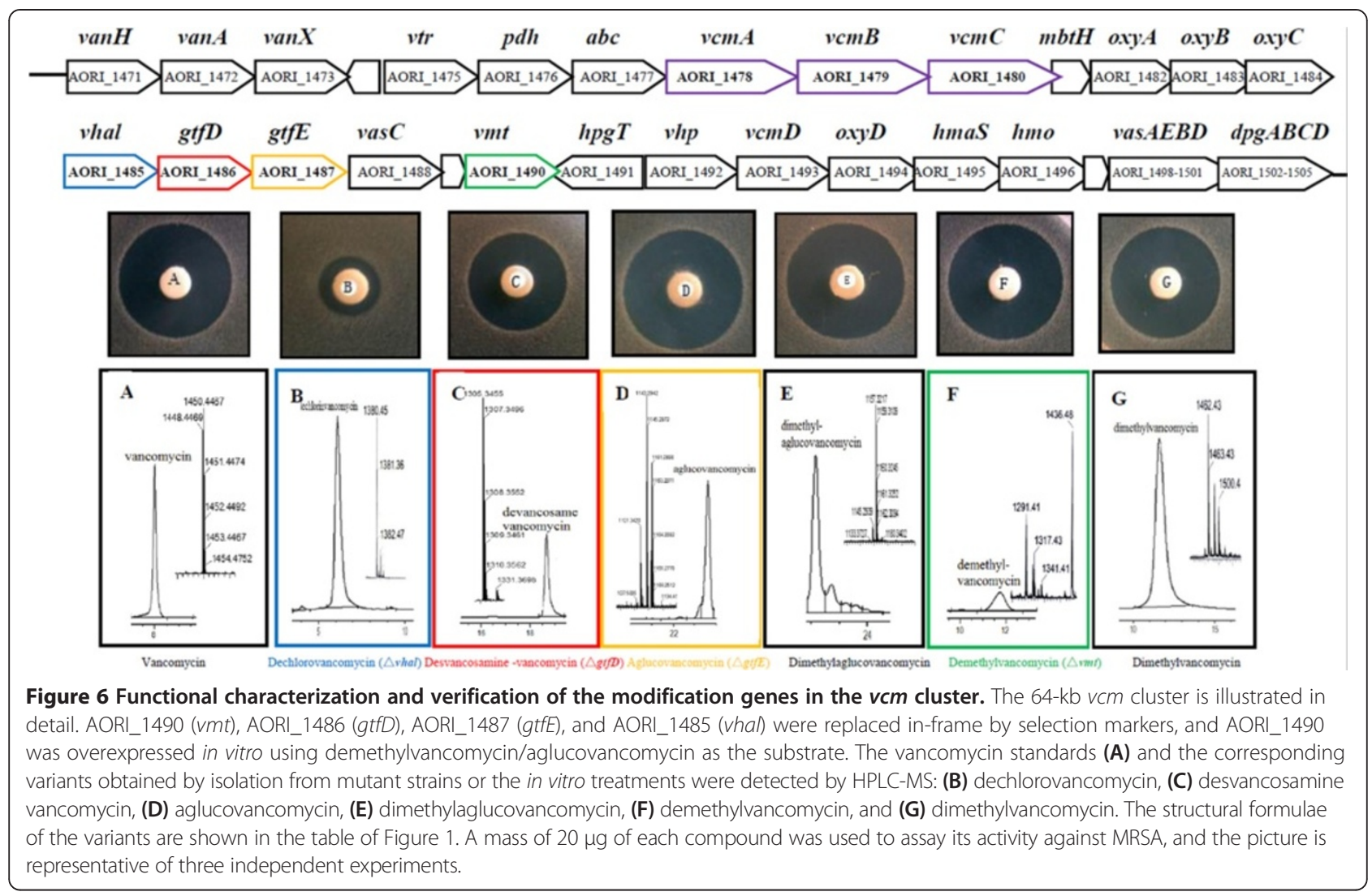

[53], indicating that the sugar moiety may play an important role in imparting enhanced pharmacokinetic properties.

With the exception of dimethylaglucovancomycin, nearly all of the vancomycin derivatives mentioned above have been isolated naturally and their antibacterial activities reported [53] and our results are in agreement with these previous findings. However, this study provides, for the first time, in vivo functional characterization of the predicted halogenase, the putative methyltransferase and the biochemically-characterized glycosyltransferases in A. orientalis, along with a systemic analysis of the distinct bioactivities of different vancomycin variants. These in vivo analyses not only demonstrated that the $v \mathrm{~cm}$ encoded halogenase and methyltransferase are functionally equivalent to those encoded in the bal and cep clusters, but also inferred that the modifications of halogenation, methylation, and glycosylation are not conducted exactly in series [17], because the vancomycin variants produced by each mutant were only deficient in their corresponding modification principally (the only exception is aglucovancomycin of the gtfE mutant, from which both the glycosyl residues were absent). In other words, the tailoring enzymes (except GtfD) are not very specific, but have broad substrate spectra in vivo.

\section{Conclusions}

The genome of $A$. orientalis HCCB10007 is the first complete sequence for the bacteria that synthesize the vancomycin group antibiotics. Compared with the phylogenetic closely related rifamycin-producing strain $A$. mediterranei, $A$. orientalis has a relatively smaller chromosome and a more compact genomic organization. Their different configurations revealed possible chromosomal recombination events representing genomic plasticity related to either vancomycin biosynthesis or rifamycin biosynthesis. By comparison with other actinomycete genomes, the common features of the Amycolatopsis genomes and molecular chemotaxonomic characteristics (MCCs) representing the phenotypes of phospholipid and menaquinone for this genus were further identified and developed. In addition, the knockout of genes encoding the tailoring enzymes in A. orientalis was achieved, and the functions of the predicted halogenase and methyltransferase annotated in the $v \mathrm{~cm}$ cluster were characterized for the first time. The data provided by this study may facilitate the development of novel lead compounds for drug development, either through a combinatorial biosynthesis approach employing enzymes with newly engineered modification activities, or using different vancomycin derivatives as the starting chemical moieties. 


\section{Methods}

\section{Genome sequencing and assembly}

A orientalis strain $\mathrm{HCCB} 10007$ was deposited in the Institute of Microbiology of Chinese Academy of Sciences and designated CGMCC No. 6023. A traditional whole genome shotgun strategy using the Roche 454 GS FLX Titanium System was applied to sequence HCCB10007's genome. In total, 53 contigs with $8.9-\mathrm{Mb}$ length were assembled from 561,423 reads (average length of $408 \mathrm{bp}$ ) by the Newbler Program (version 2.3) of the 454 suite package. The relationships between the contigs were determined by referring to the $A$. mediterranei genome or using the ContigScape plugin [54], and the remaining gaps were filled using a multiplex PCR strategy. The final sequence assembly was performed using the phred/phrap/consed package (http://www.phrap.org/phredphrapconsed.html). Sanger-based sequencing was employed to facilitate the gap closure and to amend the low-quality regions (score $<60$ ). Finally, a consensus sequence containing $8,948,591 \mathrm{bp}$ (with an estimated error rate of less than 0.5 per 100,000 bases) that provided 25.6-fold coverage was acquired.

\section{Genome annotation and analysis}

Putative protein-coding sequences were predicted based on the results from both Glimmer and Genemark. The BLASTP results obtained from the KEGG, NR, and CDD databases were used to annotate the CDSs and manual correction was also implemented. The tRNA genes were predicted directly with tRNAscan-SE v1.23. Essential genes were defined as those encoding proteins functioning in cell division, replication, transcription, translation, and amino-acid metabolism, with reference to the Clusters of Orthologous Groups (COGs) Database. Unless otherwise stated, the orthologous proteins between $A$. orientalis HCCB10007 and other related species were defined by reciprocal BLASTP under the following conditions: minimum 30\% identity and 30\% length diversity. The coding density of all of the genes was defined as the ratio of the protein-coding sequences (CDSs) length to the total genomic length, whereas the coding density of essential genes (or orthologs) was defined as the ratio of sequence length of the essential genes (or orthologs) to the total CDSs in a corresponding non-overlapping sliding window. Statistical comparisons between core $v s$. non-core and quasi-core $v s$. non-core were estimated as $P$-values calculated by the grouped $t$ test method with the statistical programming language R, employing two window sizes for analyses (except for the case of comparing the coding density of essential genes between quasi-core $v s$. non-core, $P$-values shown in the text used the 90-kb sliding window size instead of $45-\mathrm{kb}$ ). The MUMmer 3.0 Project was used to analyze the genome-wide co-linearity between $A$. orientalis HCCB10007 and A. mediterranei. To characterize the molecular chemotaxonomic characteristics (MCCs), the biosynthetic pathways of arabinose, glycine, diaminopimelic acids, mycolic acids, phospholipids, and menaquinones in $A$. orientalis and other actinomycetes were analyzed on http://www.kegg.jp/kegg/pathway.html. Literature searching, sequence alignment, domain comparison, and/or phylogenetic analysis were used to further identify the critical genes that determined the existence, conformation, and chain lengths of compounds. All of the BLASTP analyses conducted with the MCCs used a threshold E value of 1e-3. The neighbor joining (NJ) method of the MEGA 5.0 package was used to construct phylogenetic trees based on the 16S rRNA, Isp, and MurE sequences, and the reliability of each branch was tested by 1,000 bootstrap replications. For the Isp sequences, an additional maximum parsimony (MP) method was used to obtain a more robust tree topology. The SBSPKS [45] and/or NRPSDB [46] databases were used to predict the probable substrates or products for secondary metabolite biosynthetic gene clusters.

\section{Construction of monogenic mutations of AORI_1485 (vhal), AORI_1490 (vmt), AORI_1486 (gtfD) and AORI_1487 (gtfE) in A. orientalis}

A homologous recombination method similar to the PCR-targeting system applied in Streptomyces was used to mutate the vhal, vmt, $g t f D$, and $g t f E$ genes in A. orientalis HCCB10007. First, a cosmid library of $A$. orientalis genomic DNA (containing inserts of about $40 \mathrm{~Kb}$ in length) was constructed using SuperCos 1 Cosmid Vector Kit (Agilent Technologies, Inc.). The cosmid clone XL0311, which contained the AORI_1485, AORI_1490, AORI_1486, and AORI_1487 genes, was selected by Southern blotting and further used to knockout vhal, vmt, gtfD, and gtfE, individually. All of the target genes in XL0311 were replaced precisely with the apramycin-resistance gene, using two long recombinational primers (39 nt). The cosmids with mutated target genes were introduced into $E$. coli BW25113/pIJ790 ( $\lambda$ RED recombination plasmid) and then conjugated into the $A$. orientalis recipients. The correct exconjugants/knockout clones were selected based on their apramycin resistance $(50 \mu \mathrm{g} / \mathrm{ml})$ on MS medium (mannose $20 \mathrm{~g} / \mathrm{L}$, soybean flour $20 \mathrm{~g} / \mathrm{L}$, agar $20 \mathrm{~g} / \mathrm{L}$, and $10 \mathrm{mmol} / \mathrm{L} \mathrm{MgCl}_{2}$ ) supplemented with nalidixic acid $(20 \mu \mathrm{g} / \mathrm{ml})$ for counter selection. The genes inactivated by homologous recombination were further confirmed by PCR (for primers, please see Additional file 1: Supplementary Materials and Methods).

\section{Vmt expression, purification, and in vitro modification assay}

The $v m t$ gene from the A. orientalis HCCB10007 genomic DNA was cloned into a pET30a vector and transformed into BL21 (DE3) cells. The expression of $\mathrm{His}_{6}$-tagged Vmt was induced by $1 \mathrm{mM}$ isopropyl-1-thio- $\beta$-D-galactoside 
(IPTG) at $30^{\circ} \mathrm{C}$ for $4 \mathrm{~h}$, and then nickel-nitrilotriacetic acid (Ni-NTA) affinity chromatography (Qiagen, Valencia, CA, USA) was used to purify the protein. The in vitro methylation modification system contained $2 \mathrm{mM}(S)$ adenosyl-L-methionine (Sigma-Aldrich Canada), $10 \mathrm{mM}$ $\mathrm{His}_{6}$-Vmt (3 mg), $500 \mathrm{mM}$ substrate (demethylvancomycin or aglucovancomycin), and $50 \mathrm{mM}$ Tris- $\mathrm{HCl}(\mathrm{pH} 7.5)$ in a total volume of $1 \mathrm{ml}$. The reaction was conducted at $25^{\circ} \mathrm{C}$ for $24 \mathrm{~h}$. The reaction was stopped by the addition of an equal volume of cold methanol, incubated at $-20^{\circ} \mathrm{C}$ for $20 \mathrm{~min}$, and centrifuged for $5 \mathrm{~min}$ at $10,000 \times \mathrm{g}$. HPLCMS was used to analyze the supernatant.

\section{HPLC-MS analyses}

The vancomycin derivatives were prepared from ultrasonically lysed suspensions of the culture pellets, and the cell debris was removed by centrifugation and filtration. HPLC-Q-TOF-MS (Waters Micromass Q-TOF Premier Mass Spectrometer) analysis was then used to identify the derivates. HPLC was performed at $40^{\circ} \mathrm{C}$ using an ACQUITY HPLC BEH C18 column $(100 \mathrm{~mm} \times 2.1 \mathrm{~mm}$, i.d.: $1.7 \mu \mathrm{m}$; Waters Corp., Milford, USA) equipped with an ACQUITY HPLC VanGuard PreColumn $(5 \mathrm{~mm} \times 2.1 \mathrm{~mm}$, i.d.: $1.7 \mu \mathrm{m}$; Waters Corp., Milford, MA, USA). Solvent A (0.05\% TFA in water) and solvent B (0.05\% TFA in acetonitrile) were used as the mobile phase, with a flow rate of $0.4 \mathrm{ml} \mathrm{min}{ }^{-1}$. The following gradient was used: $t=0 \mathrm{~min}$ : $5 \% \mathrm{~B} ; \mathrm{t}=2.2 \min : 15 \% \mathrm{~B} ; \mathrm{t}=4.5 \mathrm{~min}: 30 \% \mathrm{~B} ; \mathrm{t}=12.5 \mathrm{~min}$ : $99 \%$ B. The mass spectrometer detected all of the samples at a wavelength of $240 \mathrm{~nm}$.

\section{Zone of inhibition test}

This test was conducted using Staphylococcus aureus cultured in LB medium as indicator cells. The soft top agar of the test agar plate consisted of $10 \mathrm{gL}^{-1}$ tryptone extract, $5 \mathrm{gL}^{-1}$ yeast extract, $5 \mathrm{gL}^{-1} \mathrm{NaCl}$, and $16 \mathrm{gL}^{-1}$ agar, and the indicator cells were added into the soft agar at a final concentration of $10^{6} \mathrm{cfu} / \mathrm{ml}$. Then, $20 \mu \mathrm{g}$ of each vancomycin variant was carefully dropped onto the drug-sensitive slips. The slips were then placed on the center of the agar plates. Observations were made after 20 hours at $37^{\circ} \mathrm{C}$. Three independent experiments were performed and the representative pictures were chosen for the figure.

\section{Nucleotide sequence accession numbers}

The nucleotide sequences of the chromosome and plasmid were deposited in the GenBank database under accession numbers [CP003410] and [CP003411], respectively.

\section{Availability of supporting data}

All the supporting data named as "Additional files for the Amycolatopsis orientalis genome paper", were deposited in an open access repository of CHGC (Chinese
National Human Genome Center at Shanghai) database. Please refer to http://chgc.sh.cn/ch/Ao.html. The phylogenetic trees based on the $16 \mathrm{~S}$ rRNA, Isp, and MurE sequences were deposited in Treebase database (www.treebase.org). Please refer to http://purl.org/phylo/treebase/phylows/study/ TB2:S15601.

\section{Additional file}

\begin{abstract}
Additional file 1: Figure S1. (A) Phylogeny tree based on $16 \mathrm{~S}$ ribosome RNA of selected actinobacteria and other related species. (B) Comparative analyses of the orthologs between different actinomycete genomes. Table S1. Comparative analysis of the $16 \mathrm{~S}$ ribosome RNAs between and in A. orientalis and A. mediterranei genomes. Table S2. The $P$-values derived from grouped t test for the coding densities of orthologs or essential genes comparing the core (or R1, or R2) region against the non-core regions under the conditions of different sliding window sizes. Table S3. Enzymes in different actinomycetes involved in the pathway of incorporating arabinose into the cell wall. Table S4. Genes characterized in different actinomycetes responsible for recruiting glycine residues crossbridging to the peptidoglycan lateral chains. Figure S2. Pylogenetic analyses of MurE in actinomycetes. Figure S3. Genetic organization of the fadD-pks-accD and fas-/ gene clusters in 20 selected actinobacterial genomes. Figure S4. phylogenetic analysis of isoprenyl diphosphate synthases from type strains of actinomycetes using the MP method. Table S5. Orthologs of secondary metabolite genes in A.orientalis HCCB10007 genome compared to the NCBI database. Figure S5. The reverse-transcription PCR of RNA isolated from different cultures. Table S6. Genes encoded for racemases in A.orientalis HCCB10007 genome. Table S7. NMR spectroscopic data for dimethylvancomycin. Table S8. NMR spectroscopic data for dimethylaglucovancomycin.
\end{abstract}

\section{Competing interests}

The authors declare that they have no competing interests.

\section{Authors' contributions}

LX carried out genome assembly, annotation, and constructed the gene mutants. $\mathrm{HH}$ isolated the bacterial strain, extracted the genomic DNA, and carried out the SEM and NMR experiments. WW carried out the protein expression, purification, and the in vitro modification assay. YZ, HY, and BT participated in genome annotation and picture drawing. LZ, MG, and SY participated in gap closure and sequence amendment. $\mathrm{WH}$ and $\mathrm{HZ}$ performed the genome sequencing. WJ performed the cosmid library construction and the zone of inhibition test experiments. DC designed and performed HPLC-MS experiments. GPZ supervised experimental work and participated in the manuscript writing. WZ designed and performed the genome analysis and draft the manuscript. All authors read and approved the final manuscript.

\section{Acknowledgements}

This work was supported by grants from the National Natural Science Foundation of China (30672558, 30772678, 30830002, 31121001, 31270056), the National Basic Research Program of China (2012CB721102, 2013CB531603), the Postdoctoral Science Foundation of China (2012 M510787) and the National S\&T Major Special Project on New Drug Innovation (2009ZX9302-004).

\section{Author details}

${ }^{1}$ Nanjing Agricultural University, Nanjing 210095, China. ${ }^{2}$ Shanghai Laiyi Center for Biopharmaceutical R\&D, Shanghai 200240, China. ${ }^{3}$ CAS Key Laboratory of Synthetic Biology, Institute of Plant Physiology and Ecology, Shanghai Institutes for Biological Sciences, Chinese Academy of Sciences, Shanghai 200031, China. "State Key Laboratory of Genetic Engineering, Department of Microbiology, School of Life Sciences and Institute of Biomedical Sciences, Fudan University, Shanghai 200433, China.

${ }^{5}$ Shanghai-MOST Key Laboratory of Disease and Health Genomics, Chinese National Human Genome Center at Shanghai, Shanghai 201203, China. ${ }^{6}$ Shanghai Institute of Pharmaceutical Industry, Shanghai 200040, China. 
${ }^{7}$ Department of Microbiology and Li Ka Shing Institute of Health Sciences, The Chinese University of Hong Kong, Prince of Wales Hospital, Shatin, New Territories, Hong Kong, SAR, China. ${ }^{8}$ China HKY Gene Technology Company Ltd, Shenzhen, Guangdong 518057, China. ' Medical College, Shenzhen University, Shenzhen, Guangdong 518060, China. ${ }^{10}$ Current address: Computational Biology Center, Memorial Sloan Kettering Cancer Center, New York, NY 10065, USA

Received: 7 November 2013 Accepted: 14 April 2014

Published: 13 May 2014

\section{References}

1. Foldes M, Munro R, Sorrell TC, Shanker S, Toohey M: In-vitro effects of vancomycin, rifampicin, and fusidic acid, alone and in combination, against methicillin-resistant Staphylococcus aureus. J Antimicrob Chemother 1983, 11(1):21-26.

2. Walsh CT, Fisher SL, Park IS, Prahalad M, Wu Z: Bacterial resistance to vancomycin: five genes and one missing hydrogen bond tell the story. Chem Biol 1996, 3(1):21-28.

3. Chang S, Sievert DM, Hageman JC, Boulton ML, Tenover FC, Downes FP, Shah S, Rudrik JT, Pupp GR, Brown WJ, Cardo D, Fridkin SK: Infection with vancomycin-resistant Staphylococcus aureus containing the vanA resistance gene. N Engl J Med 2003, 348(14):1342-1347.

4. Chambers HF, Deleo FR: Waves of resistance: Staphylococcus aureus in the antibiotic era. Nat Rev Microbiol 2009, 7(9):629-641.

5. Calfee DP: Methicillin-resistant Staphylococcus aureus and vancomycinresistant enterococci, and other Gram-positives in healthcare. Curr Opin Infect Dis 2012, 25(4):385-394.

6. Guskey MT, Tsuji BT: A comparative review of the lipoglycopeptides: oritavancin, dalbavancin, and telavancin. Pharmacotherapy 2010, 30(1):80-94

7. Van Wageningen AM, Kirkpatrick PN, Williams DH, Harris BR, Kershaw JK, Lennard NJ, Jones M, Jones SJ, Solenberg PJ: Sequencing and analysis of genes involved in the biosynthesis of a vancomycin group antibiotic. Chem Biol 1998, 5(3):155-162.

8. Pelzer S, Sussmuth R, Heckmann D, Recktenwald J, Huber P, Jung G, Wohlleben W: Identification and analysis of the balhimycin biosynthetic gene cluster and its use for manipulating glycopeptide biosynthesis in Amycolatopsis mediterranei DSM5908. Antimicrob Agents Chemother 1999, 43(7):1565-1573

9. Puk O, Huber P, Bischoff D, Recktenwald J, Jung G, Sussmuth RD, van Pee KH, Wohlleben W, Pelzer S: Glycopeptide biosynthesis in Amycolatopsis mediterranei DSM5908: function of a halogenase and a haloperoxidase/ perhydrolase. Chem Biol 2002, 9(2):225-235.

10. Losey HC, Peczuh MW, Chen Z, Eggert US, Dong SD, Pelczer I, Kahne D, Walsh CT: Tandem action of glycosyltransferases in the maturation of vancomycin and teicoplanin aglycones: novel glycopeptides. Biochemistry 2001, 40(15):4745-4755.

11. Mulichak AM, Losey HC, Walsh CT, Garavito RM: Structure of the UDPglucosyltransferase GtfB that modifies the heptapeptide aglycone in the biosynthesis of vancomycin group antibiotics. Structure 2001, 9(7):547-557.

12. Mulichak AM, Losey HC, Lu W, Wawrzak Z, Walsh CT, Garavito RM: Structure of the TDP-epi-vancosaminyltransferase GtfA from the chloroeremomycin biosynthetic pathway. Proc Natl Acad Sci U S A 2003, 100(16):9238-9243.

13. Lu W, Oberthur M, Leimkuhler C, Tao J, Kahne D, Walsh CT: Characterization of a regiospecific epivancosaminyl transferase GtfA and enzymatic reconstitution of the antibiotic chloroeremomycin. Proc Natl Acad Sci U S A 2004, 101(13):4390-4395.

14. Shi R, Lamb SS, Zakeri B, Proteau A, Cui Q, Sulea T, Matte A, Wright GD, Cygler M: Structure and function of the glycopeptide Nmethyltransferase MtfA, a tool for the biosynthesis of modified glycopeptide antibiotics. Chem Biol 2009, 16(4):401-410.

15. Losey HC, Jiang J, Biggins JB, Oberthur M, Ye XY, Dong SD, Kahne D, Thorson JS, Walsh CT: Incorporation of glucose analogs by GtfE and GtfD from the vancomycin biosynthetic pathway to generate variant glycopeptides. Chem Biol 2002, 9(12):1305-1314.

16. Solenberg PJ, Matsushima P, Stack DR, Wilkie SC, Thompson RC, Baltz RH: Production of hybrid glycopeptide antibiotics in vitro and in Streptomyces toyocaensis. Chem Biol 1997, 4(3):195-202.
17. Hubbard BK, Walsh $C T$ : Vancomycin assembly: nature's way. Angew Chem Int Ed Engl 2003, 42(7):730-765.

18. Basch J, Chiang SJ: Cloning and expression of a cytochrome P450 hydroxylase gene from Amycolatopsis orientalis: hydroxylation of epothilone B for the production of epothilone F. J Ind Microbiol Biotechnol 2007, 34(2):171-176.

19. Zerbe K, Pylypenko O, Vitali F, Zhang W, Rouset S, Heck M, Vrijbloed JW, Bischoff D, Bister B, Sussmuth RD, Pelzer S, Wohlleben W, Robinson JA, Schlichting I: Crystal structure of OxyB, a cytochrome P450 implicated in an oxidative phenol coupling reaction during vancomycin biosynthesis. J Biol Chem 2002, 277(49):47476-47485.

20. Marshall CG, Broadhead G, Leskiw BK, Wright GD: D-Ala-D-Ala ligases from glycopeptide antibiotic-producing organisms are highly homologous to the enterococcal vancomycin-resistance ligases VanA and VanB. Proc Natl Acad Sci U S A 1997, 94(12):6480-6483.

21. Marshall CG, Lessard IA, Park I, Wright GD: Glycopeptide antibiotic resistance genes in glycopeptide-producing organisms. Antimicrob Agents Chemother 1998, 42(9):2215-2220.

22. Jeong H, Sim YM, Kim HJ, Lee YJ, Lee DW, Lim SK, Lee SJ: Genome sequences of Amycolatopsis orientalis subsp. orientalis strains DSM 43388 and DSM 46075. Genome Announcements 2013, 1:4.

23. Jeong H, Sim YM, Kim HJ, Lee DW, Lim SK, Lee SJ: Genome sequence of the vancomycin-producing Amycolatopsis orientalis subsp. orientalis strain KCTC 9412T. Genome Announcements 2013, 1:3.

24. Zhao W, Zhong $Y$, Yuan $H$, Wang J, Zheng $H$, Wang $Y$, Cen $X$, Xu F, Bai J, Han X, Lu G, Zhu Y, Shao Z, Yan H, Li C, Peng N, Zhang Z, Zhang Y, Lin W, Fan Y, Qin Z, Hu Y, Zhu B, Wang S, Ding X, Zhao GP: Complete genome sequence of the rifamycin SV-producing Amycolatopsis mediterranei U32 revealed its genetic characteristics in phylogeny and metabolism. Cell Res 2010, 20(10):1096-1108.

25. Tang B, Zhao W, Zheng H, Zhuo Y, Zhang L, Zhao GP: Complete genome sequence of Amycolatopsis mediterranei $\mathbf{S 6 9 9}$ based on de novo assembly via a combinatorial sequencing strategy. J Bacteriol 2012, 194(20):5699-5700

26. Verma M, Kaur J, Kumar M, Kumari K, Saxena A, Anand S, Nigam A, Ravi V, Raghuvanshi S, Khurana P, Tyagi AK, Khurana JP, Lal R: Whole genome sequence of the rifamycin B-producing strain Amycolatopsis mediterranei S699. J Bacteriol 2011, 193(19):5562-5563.

27. Ohnishi Y, Ishikawa J, Hara H, Suzuki H, Ikenoya M, Ikeda H, Yamashita A, Hattori M, Horinouchi S: Genome sequence of the streptomycinproducing microorganism Streptomyces griseus IFO 13350. J Bacteriol 2008, 190(11):4050-4060

28. Davis JR, Goodwin LA, Woyke T, Teshima H, Bruce D, Detter C, Tapia R, Han S, Han J, Pitluck S, Nolan M, Mikhailova N, Land ML, Sello JK: Genome sequence of Amycolatopsis sp. strain ATCC 39116, a plant biomassdegrading actinomycete. J Bacteriol 2012, 194(9):2396-2397.

29. Aziz RK, Bartels D, Best AA, DeJongh M, Disz T, Edwards RA, Formsma K, Gerdes S, Glass EM, Kubal M, Meyer F, Olsen GJ, Paarmann D, Paczian T, Parrello B, Pusch GD, Reich C, Stevens R, Vassieva O, Vonstein V, Wilke A, Zagnitko O: The RAST Server: rapid annotations using subsystems technology. BMC Genomics 2008, 9:75.

30. Bentley SD, Chater KF, Cerdeno-Tarraga AM, Challis GL, Thomson NR, James KD, Harris DE, Quail MA, Kieser H, Harper D, Bateman A, Brown S, Chandra G, Chen CW, Collins M, Cronin A, Fraser A, Goble A, Hidalgo J, Hornsby T, Howarth S, Huang CH, Kieser T, Larke L, Murphy K, Oliver S, O'Neil S, Rabbinowitsch E, Rajandream MA, Rutherford K, et al: Complete genome sequence of the model actinomycete Streptomyces coelicolor A3(2). Nature 2002, 417(6885):141-147.

31. Oliynyk M, Samborskyy M, Lester JB, Mironenko T, Scott N, Dickens S, Haydock SF, Leadlay PF: Complete genome sequence of the erythromycin-producing bacterium Saccharopolyspora erythraea NRRL23338. Nat Biotechnol 2007, 25(4):447-453.

32. Pittenger RC, Brigham RB: Streptomyces orientalis $\mathrm{n}$. sp., the source of vancomycin. Antibiot 1956, 6:642-647.

33. Thiemann JE, Zucco G, Pelizza G: A proposal for the transfer of Streptomyces mediterranei Margalith and Beretta 1960 to the genus Nocardia as Nocardia mediterranea (Margalith and Beretta) comb. nov. Arch Mikrobiol 1969, 67(2):147-155.

34. Lechevalier MP, Prauser H, Labeda DP, Ruan JS: Two new genera of nocardioform actinomycetes: amycolata gen.nov. and Amycolatopsis gen. nov. Int J Syst Bacteriol 1986, 36(1):29-37. 
35. Mary P, Lechevalier CDB, Hubert L: Chemotaxonomy of aerobic Actinomycetes: phospholipid composition. Biochem Syst Ecol 1977, 5(4):249-260.

36. Ishinaga M, Kito M: Participation of soluble phosphatidylserine synthetase in phosphatidylethanolamine biosynthesis in Escherichia coli membrane. Eur J Biochem/FEBS 1974, 42(2):483-487.

37. Kanfer J, Kennedy EP: Metabolism and function of bacterial lipids. II. Biosynthesis of phospholipids in Escherichia Coli. J Biol Chem 1964 239:1720-1726.

38. Nowicka B, Kruk J: Occurrence, biosynthesis and function of isoprenoid quinones. Biochim Biophys Acta 2010, 1797(9):1587-1605

39. Olsen I: Chemotaxonomy of Bacteroides: a review. Acta Odontol Scand 1994, 52(6):354-367.

40. Blinov NO, lakubov GZ, Sokolova NL, Khokhlov AS: Classification of antibiotics-quinones of actinomycetic origin. Izvestiia Akademii nauk SSSR Seriia biologicheskaia 1967, 3:357-373.

41. Wang K, Ohnuma S: Chain-length determination mechanism of isoprenyl diphosphate synthases and implications for molecular evolution. Trends Biochem Sci 1999, 24(11):445-451.

42. Zhi XY, Zhao W, Li WJ, Zhao GP: Prokaryotic systematics in the genomics era. Antonie Van Leeuwenhoek 2012, 101(1):21-34.

43. Udwary DW, Zeigler L, Asolkar RN, Singan V, Lapidus A, Fenical W, Jensen PR, Moore BS: Genome sequencing reveals complex secondary metabolome in the marine actinomycete Salinispora tropica. Proc Nat Acad Sci U S A 2007, 104(25):10376-10381.

44. Banskota AH, McAlpine JB, Sorensen D, Ibrahim A, Aouidate M, Piraee M, Alarco AM, Farnet CM, Zazopoulos E: Genomic analyses lead to novel secondary metabolites. Part 3. ECO-0501, a novel antibacterial of a new class. J Antibiot (Tokyo) 2006, 59(9):533-542.

45. Anand S, Prasad MV, Yadav G, Kumar N, Shehara J, Ansari MZ, Mohanty D: SBSPKS: structure based sequence analysis of polyketide synthases. Nucleic Acids Res 2010, 38(Web Server issue):W487-W496.

46. Ansari MZ, Yadav G, Gokhale RS, Mohanty D: NRPS-PKS: a knowledgebased resource for analysis of NRPS/PKS megasynthases. Nucleic Acids Res 2004, 32(Web Server issue):W405-W413.

47. Trauger JW, Walsh CT: Heterologous expression in Escherichia coli of the first module of the nonribosomal peptide synthetase for chloroeremomycin, a vancomycin-type glycopeptide antibiotic. Proc Natl Acad Sci U S A 2000, 97(7):3112-3117.

48. Rausch C, Hoof I, Weber T, Wohlleben W, Huson DH: Phylogenetic analysis of condensation domains in NRPS sheds light on their functional evolution. BMC Evol Biol 2007, 7:78.

49. Balibar CJ, Vaillancourt FH, Walsh CT: Generation of D amino acid residues in assembly of arthrofactin by dual condensation/epimerization domains. Chem Biol 2005, 12(11):1189-1200

50. Cava F, Lam H, de Pedro MA, Waldor MK: Emerging knowledge of regulatory roles of D-amino acids in bacteria. Cell Mol Life Sci CMLS 2011 , 68(5):817-831.

51. Woithe K, Geib N, Zerbe K, Li DB, Heck M, Fournier Rousset S, Meyer O, Vitali F, Matoba N, Abou Hadeed K, Robinson JA: Oxidative phenol coupling reactions catalyzed by OxyB: a cytochrome P450 from the vancomycin producing organism. implications for vancomycin biosynthesis. J Am Chem Soc 2007, 129(21):6887-6895.

52. Puk O, Bischoff D, Kittel C, Pelzer S, Weist S, Stegmann E, Sussmuth RD, Wohlleben W: Biosynthesis of chloro-beta-hydroxytyrosine, a nonproteinogenic amino acid of the peptidic backbone of glycopeptide antibiotics. J Bacterio/ 2004, 186(18):6093-6100.

53. Nagarajan R: Structure-activity relationships of vancomycin-type glycopeptide antibiotics. J Antibiot (Tokyo) 1993, 46(8):1181-1195.

54. Tang B, Wang Q, Yang M, Xie F, Zhu Y, Zhuo Y, Wang S, Gao H, Ding X, Zhang L, Zhao G, Zheng H: ContigScape: a Cytoscape plugin facilitating microbial genome gap closing. BMC Genomics 2013, 14:289.

doi:10.1186/1471-2164-15-363

Cite this article as: Xu et al:: Complete genome sequence and comparative genomic analyses of the vancomycin-producing Amycolatopsis orientalis. BMC Genomics 2014 15:363.

\section{Submit your next manuscript to BioMed Central and take full advantage of:}

- Convenient online submission

- Thorough peer review

- No space constraints or color figure charges

- Immediate publication on acceptance

- Inclusion in PubMed, CAS, Scopus and Google Scholar

- Research which is freely available for redistribution

Submit your manuscript at www.biomedcentral.com/submit
C) Biomed Central 